DE92 002592

\title{
TECHNICAL PROGRESS REPORT of the
}

\section{INDIANA UNIVERSITY HIGH ENERGY PHYSICS GROUP, Task C}

\author{
January 1, 1991 - December 31, 1991 \\ Richard M. Heinz, Stuart L. Mufson, and James Musser \\ Department of Physics \\ Indiana University \\ Bloomington, Indiana 47405
}

The Indiana University High Energy Physics Group, Task $C$ has been actively involved in the MACRO experiment at Gran Sasso and the SSC experiment $L^{\star}$ during the current contract year. MACRO is a large US-Italian $\underline{M}$ onopole, $\underline{A}$ strophysics, and $\underline{C}$ osmic $\underline{R}$ ay $\underline{O}$ bservatory being built under the Gran Sasso Mountain outside of Rome. Indiana University is in charge of organizing the United States software effort. We have built a state-of-the-art two-meter spectrophotometer for the MACRO liquid scintillator. We are in charge of ERP, the MACRO Event Reconstruction Processor online trigger processor for muons and stellar collapse. We are designing an air Cerenkov array to be placed on top of the Gran Sasso. Our other activity involves participation in the SSC experiment $L^{\star}$. As long-standing members of $L^{\star}$ we have done proposal writing and have worked on important $\mathrm{L}^{\star}$ planning and organization matters. We are now doing development work on the $L^{\star}$ Central Tracker straw drift tubes, including gas optimization, readout, and Monte Carlos.

* Supported by the Department of Energy Contract DOE-AC02-84ER40125 Task C.

\section{NOTICE}

This report was prepared as an account of work sponsored by the United States Government. Neither the United States nor the United States Department of Energy, nor any of their employees, nor any of their contractors, subcontractors, or their employees, makes any warranty, express or implied, or assumes any legal liability or responsibility for the accuracy, completeness, or usefulness of any information, apparatus, product or process disclosed or represents that its use would not infringe on privately owned rights.

\section{DISCLAIMER}

\footnotetext{
This report was prepared as an account of work sponsored by an agency of the United States Government. Neither the United States Government nor any agency thereof, nor any of their employees, makes any warranty, express or implied, or assumes any legal liability or responsibility for the accuracy, completeness, or usefulness of any information, apparatus, product, or process disclosed, or represents that its use would not infringe privately owned rights. Reference herein to any specific commercial product, process, or service by trade name, trademark, manufacturer, or otherwise does not necessarily constitute or imply its endorsement, recommendation, or favoring by the United Siates Guvernment or añy agency therét. The views and opinions of authors expressed herein do not necessarily state or reflect those of the United States Government or any agency thereof.
} 


\section{Table of Contents}

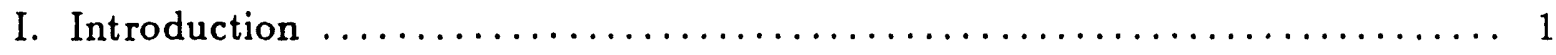

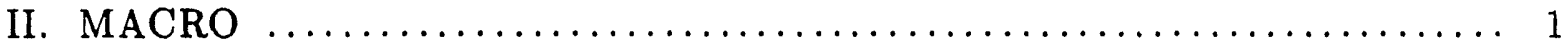

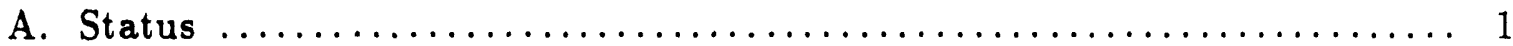

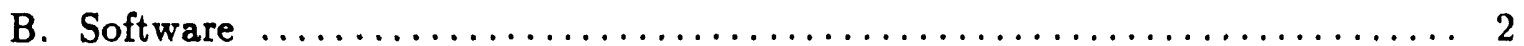

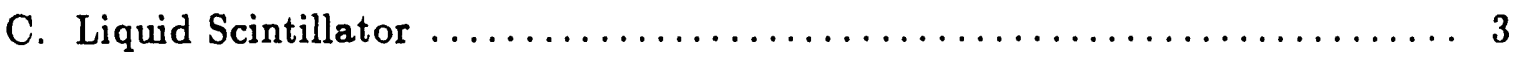

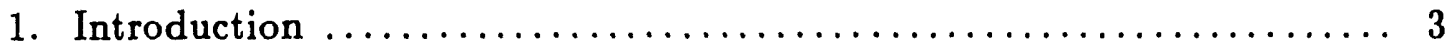

2. Oil $\ldots \ldots \ldots \ldots \ldots \ldots \ldots \ldots \ldots \ldots \ldots \ldots \ldots \ldots \ldots \ldots \ldots \ldots \ldots \ldots \ldots \ldots \ldots, 4$

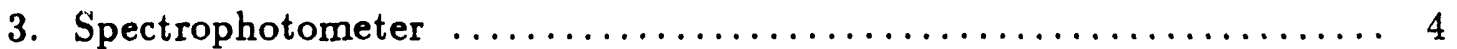

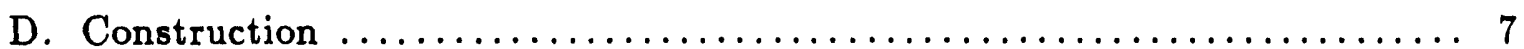

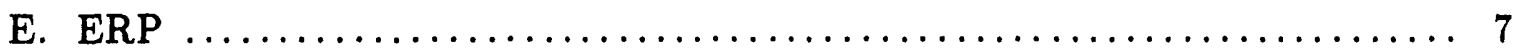

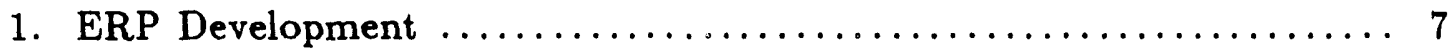

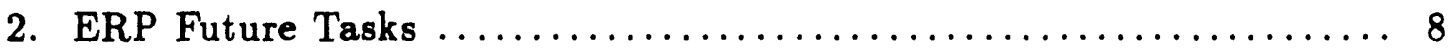

3. Scientific Objectives of Gravitational Collapse Upgrade ........... 9

4. Characteristics of a Stellar Collapse Observation in MACRO ....... 11

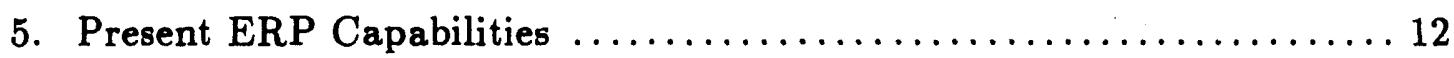

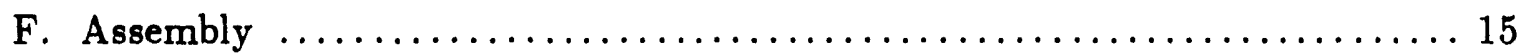

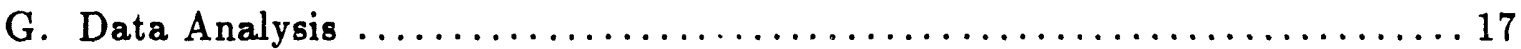

1. MACRO as a Muon Telescope $\ldots \ldots \ldots \ldots \ldots \ldots \ldots \ldots \ldots \ldots \ldots \ldots \ldots$

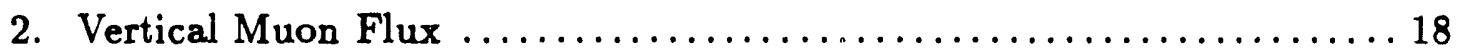

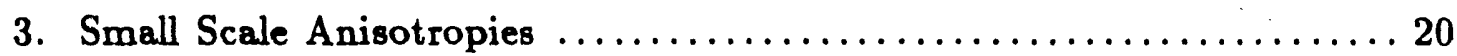

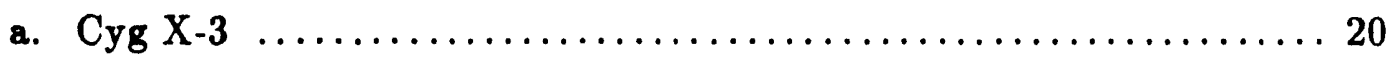

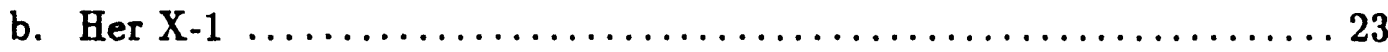

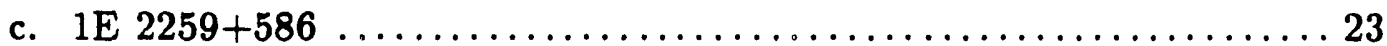

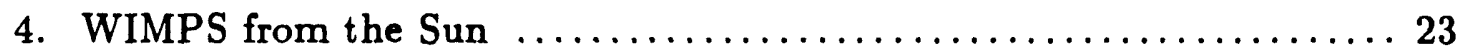

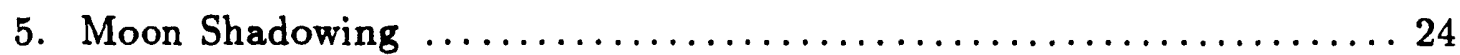

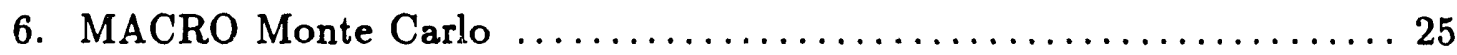

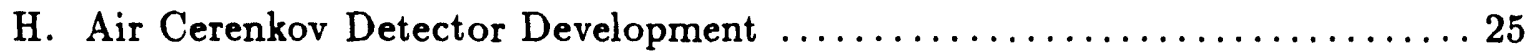

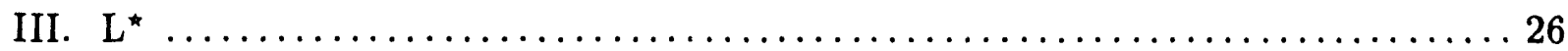




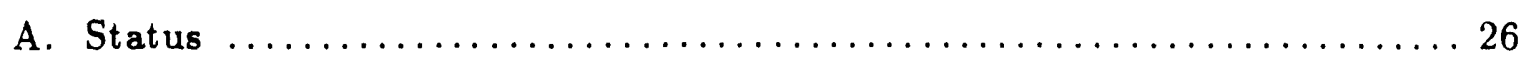

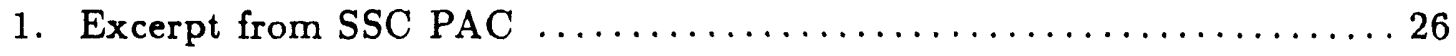

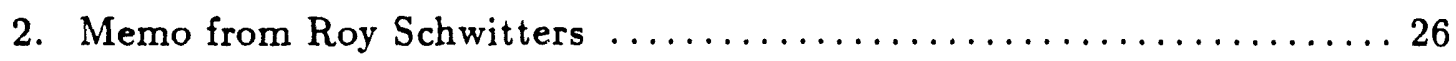

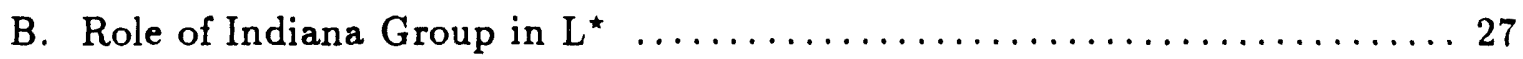

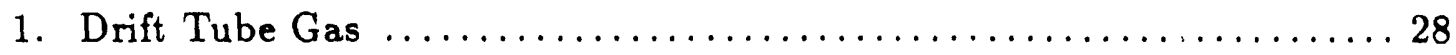

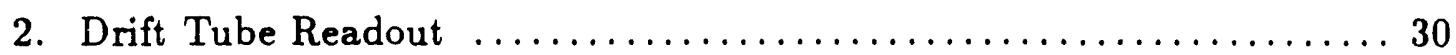

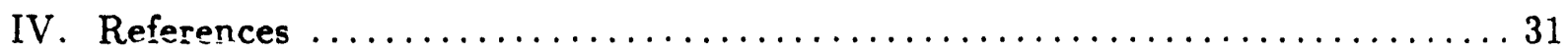

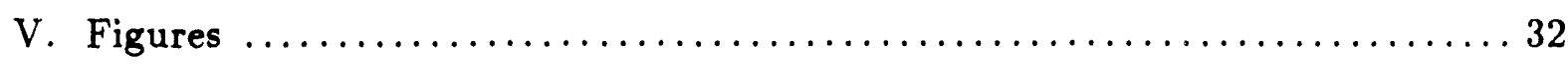




\section{Introduction}

Our Indiana Task C group consists of three faculty (Richard M. Heinz, Stuart L. Mufson, and James Musser), an Associate Scientist (Chuck Bower) a postdoc (Scott Nutter), a programmer (George Turner), four technicians (Mark Gebhard, Ersilia Giusti, James Pitts, and Mike Yeakey), and four graduate students (Alec Habig, Alice Hawthorne, Alex Komives, and Lynn Miller). We are participating in the experiments MACRO at the Gran Sasso and $\mathrm{L}^{\star}$ at the SSC.

A collaboration of US and Italian physicists is building MACRO, a large $M$ onopole, $\underline{A}$ strophysics, and $\underline{C}$ osmic $\underline{R}$ ay $\underline{O}$ bservatory, under the Gran Sasso Mountain outside of Rome. Our completion date should be early in 1993. The MACRO experiment will be optimized to search for GUT magnetic monopoles (mass $10^{16} \mathrm{GeV}$ ) in the cosmic rays with a sensitivity which is an order of magnitude below the Parker limit of $10^{-1 \mathrm{~s}} / \mathrm{cm}^{2} / \mathrm{s} / \mathrm{sr}$. Additional scientific goals include searches for extraterrestrial neutrinos, both as periodic emission from binary sources and as bursts from collapsing stars. The experiment will also use the earth as an oscillation medium to explore neutrino oscillations in the $10^{-3} \mathrm{eV}$ neutrino mass region. We will do cosmic ray composition measurements. Finally, we will search for new forms of matter emanating from sonrces such as Cygnus X-3.

We discuss in Section II the status of MACRO, the MACRO work we have done and are doing, and some MACRO results from a part of the MACRO detector. These early MACRO data were used by one of our students, John Petrakis, to finish his PhD in December, 1990. His thesis was entitled "A Search for Extraterrestrial Point Sources of Cosmic Ray Muons Using the MACRO detector." We also used these data for two MACRO publications [1](2)

$L^{\star}$ is a $4 \pi$ solenoidal magnet detector which emphasizes precise measurements of jets, electrons, muons, and photons. In $L^{\star}$ we will search for the Higgs Boson, the charged Higgs excited W's, excited Z's and the Top Quark. By measuring photons, electrons, muons, and jets with high resolution we will be sensitive to other new phenomena which will inevitably be accessibily at SSC energies, but which are not anticipated in the Standard Model or in existing extensions to the Standard Model. Section III will describe the $L^{\star}$ status and our $\mathrm{L}^{\star}$ activities.

\section{MACRO}

\section{A. Status}

MACRO is a massive detector utilizing liquid scintillator, streamer tubes, and tracketch detectors. It is organized into 12 supermodules, each $12 \mathrm{~m}$ by $12 \mathrm{~m}$ by $4.5 \mathrm{~m}$ high. As shown in Figure 1, the supermodules are arranged in a double-decker $12 \mathrm{~m}$ wide by $72 \mathrm{~m}$ long. (A detailed description of MACRO has been given in previous Progress Reports and in the MACRO proposal.) 
The status of the MACRO detector construction as of April 1, 1991 is as follows.

The prototype supermodule, SM1, is fully equipped and operational. The initial SM1 engineering test runs occurred between February 27, 1989 and May 31, 1989. Operations were resumed in the fall of 1989 , and SM1 has been running nearly continuously since then. As more of the supermodules become completely equipped and are put into the data acquistion mode, several of the electronics components of SM1 will be reworked and updated to reflect the improvements developed during the shakedown period.

SM2 has been operational, but currently is down while the scintillator tanks that are stacked immediately on top of each other (vertical tanks) receive a modification of photomultiplier (PMT) bases. The vertical tank bases have to be changed because they were designed for a higher operating voltage than has been settled on. When we discovered that SM2's PMT's were sensitive to the earth's magnetic field, using a higher voltage was one proposed solution, and the bases for SM2 verticals were designed accordingly. After the adoption of a magnetic shield solution, a lower voltage was suitable and more desirable because the dynamic range is better. Testing of the SM2 trigger electronics and the PMT's is currently underway.

SM3,4,5 are physically (but not electronically) complete. The final electronics commissioning will occur as soon as the fanouts for the various triggers arrive. SM3 and SM4 were brought into the online data acquisition system on February, 1991.

SM6 - Installation of magnetic shields and PMT's is currently under way. SM6 needs fanouts, PMT bases, and cabling.

Nearly all of the 290 scintillator tanks in the first six supermodules have been filled with the mineral oil/scintillant mixture, the exceptions being the five tanks with unrepaired leaks.

We plan to construct the remaining six MACRO supermodules (SM7-12, the Attico) by 1992 , and to have the completed MACRO detector routinely taking data in early 1993 . We will continue to take data as we build the rest of the detector. With SM1-4 operational our muon trigger rate is $.13 \mathrm{~Hz}$, or about 2 muons per $S M$ per minute.

We have accumulated a significant amount of data from SM1 and, to a lesser degree, SM2. The two millionth muon event was recorded by MACRO on March 25, 1991.

\section{B. Software}

As Mufson is the coordinator of the Offline Analysis software for the U.S., we are currently developing a body of code to meet the offline needs of the experiment. In particular, our attention is currently focused on the production of Data Summary Tapes (= DST tapes) and the further development of routines which will provide decoding (PASS $=1$ ) and the implementation of routines for muon and neutrino astronomy. 
These routines, which we are currently developing in collaboration with the Italians for implementation in the external lab computer (VAXGS), have been given the acronym

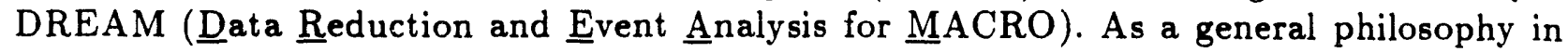
the development of the program code, we have decided to use standard libraries as widely as possible. In particular, the data structures created by DREAM will be managed by ZEBRA and will be accessed by the FZ and RZ input/output packages, where ZEBRA, FZ, and RZ are available from the CERN Program Library. In addition, the CERN HBOOK, HPLOT, PAW and GKS graphics will be used for histogramming and graphics output.

Raw data coming from the online data acquisition system are stored onto disks of the online computer VXMACR. Raw data tapes are presently being written at the rate of approximately four tapes per week (equivalent to $\sim 1$ exabyte/month). Soon the data rate will double once again when data from supermodules 3 and 4 become available.

In the second pass of DREAM (PASS $=2$ ), routines for basic data analysis functions, such as track fitting from the streamer tube hits, are also included. By running DREAM on the Storage Tapes (= ST tapes) created by PASS $=1$ at the user's home institution, this additional information will be appended to the data records. Routines for further data analysis, such as the search for astrophysical point sources, are currently being developed. Standardized routines will not be implemented as a general strategy. Until a significant number of events have been analyzed, DREAM will not filter events in the ST tapes.

In addition to the production of ST tapes, the DREAM program will include many other utilities for data analysis. Among these are the calibration of data. Among our tasks during 1991, we have incorporated calibration routines for the ERP into DREAM.

\section{Liquid Scintillator}

\section{Introduction}

The MACRO liquid scintillator mix consists of one part of a concentrate (an aromatic hydrocarbon, a wavelength shifter, and a deoxidant) and approximately 19 parts of mineral oil. MACRO will use approximately one million liters of mineral oil in brewing the liquid scintillator. The scintillator cost will be about $\$ 1 /$ liter. Our work during the current contract year includes the ongoing checks of mineral oil quality, mixing the oil into scintillator at Frascati, preparing the PVC tanks at the Gran Sasso for the scintillator, and filling these PVC tanks. We have also designed and constructed a spectrophotometer with a $2 \mathrm{~m}$ cell length. 


\section{Oil}

We check the oil quality prior to shipping from Perth Amboy, New Jersey and after arrival in Italy. (We are not commited to accepting the oil unless both checks are satisfactory.) This is done, primarily, by measuring the oil attenuation length. (We also do visual checks using a microscope and we measure the index of refraction.) The attenuation measurement is difficult because we must measure a long attenuation length at about 420 nm.

Commercial spectrophotometers have a maximum cell size of $10 \mathrm{~cm}$, too short for measurements of attenuation lengths over $20 \mathrm{~m}$. (MACRO oil has an oil attenuation length $\lambda$ of $20 \mathrm{~m}$ to $30 \mathrm{~m}$.) Therefore, in 1988 we developed a spectrophotometer with a $1 \mathrm{~m}$ long cell. We built two such spectrophotometers and use one in Bloomington and one in Frascati. These spectrophotometers are ultra sensitive to alignment and mechanical instabilities. They are not user-friendly. Thus we have designed a new spectrophotometer as discussed below.

We accepted shipments of 154,400 liters of oil in late 1990 through March, 1991. We have now filled Supermodules 1-6, except for five (leaking) tanks out of 290.

\section{Spectrophotometer}

It has long been known that stringent quality control of the large volumes of mineral oil used in the MACRO experiment is crucial for proper operation of this vast detector. The clarity to photons in the region of $420 \mathrm{~nm}$, the peak of the emission region of the wavelength shifter mixed into this oil, is of primary interest. Our group has taken considerable effort to provide a method for repeatable characterization of this clarity. This work has also resulted in a technique for measuring the clarity of materials other than mineral oil as an added bonus.

The clarity of a material is specified by its attenuation length. The attenuation length $\lambda$ is defined by $I=I_{0} \times \exp (-x / \lambda)$, where $I$ is the light intensity after an initial intensity $I_{\circ}$ propagates a distance $x$.

This implies that the attenuation length is simply the distance that light must travel to drop $63 \%$ in intensity. For some materials such as silica glass, this distance could be as little as 8 meters. Ordinary tap water could reach an attenuation length of 10 meters, while highly purified water that has been distilled and deionized can have values as long as 40 meters. The mineral oils used in the MACRO experiment have attenuation lengths in the range of 20-30 meters.

To help in the characterization of this mineral oil, Indiana University designed a spectrophotometer with a two-meter cell. A schematic of this device is presented in Figure 2. The operation of such a device in principle is very simple. Light from a laser of peak emission $420 \mathrm{~nm}$ is split into two equal components. One of these beams, called the primary 
beam, is sent through a PVC (polyvinyl chloride) tube filled with an oil sample. This tube is labelled Sample Tube in Figure 2. After being attenuated by the oil, the beam hits the face of a photomultiplier tube. The second beam, called the secondary, is bounced over a long path length to provide a time delay from the primary before being sent to the same photomultiplier tube. The signals are stored on a digital oscilloscope.

This method for measuring an attenuation length of a substance is straightforward. First, the time integral of the signals from the primary and secondary beams are measured for a run with the two-meter long sample target in the system. This process is repeated with a target that has the same windows as the sample target (quartz), but is very thin and contains a small sample of the material being measured. This very thin target is designed to simulate the light losses due to reflections at the interface of the window to the air in the room and to the sample in the target tube without having any appreciable loss due to the attenuation length of the oil.

Since the secondary beam is not affected by the sample target measurement or the thin target measurement occurring in the primary beam, it can be used as a reference. The ratio of primary to secondary for both runs is directly related to the attenuation length $\lambda$ by $\lambda=-L / \ln \left(r / r_{\text {out }}\right)$ where $L$ is the length of the sample tube, $r$ is Sample Tube ratio, and $r_{\text {out }}$ is the ratio for the thin target.

The basic hardware used in this spectrophotometer starts with the laser. This unit is capable of delivering repeated packets of very fast and intense light. A coumaran die module placed on the front of this unit restricts the light to the region of $420 \mathrm{~nm}$. The pulse repetition rate is set on the laser, and can take on a value from $1-20 \mathrm{~Hz}$. The laser has excellent timing characteristics with a mean pulse width of less than $3 \mathrm{~ns}$, during which time a signal of 120 microjoules of delivered.

The photomultiplier tube which measures both the primary and secondary beam is an Amperex XP2020. This tube has excellent timing characteristics with a mean pulse width of roughly 20 ns. Pulse height resolutions for these tubes are in the range of $6-10 \%$.

The targets for the spectrophotometer are very simple in nature. The sample target tube is two meters in length and is made of ordinary PVC. At each end of the tube is a quartz window held to the PVC with RTV sealant. A small hole in the side of the tube allows for both filling of the tube with the sample and the cleaning of the tube between samples. The thin target uses two quartz windows (as are used in the long target) placed on both sides of a ring of PVC. The gap between these quartz faces is on the order of 3 $\mathrm{mm}$. A small hole in the PVC ring permits filling of the thin target with a syringe and cleaning between samples.

The remainder of the system is composed of optics. Mirrors used to direct the primary and secondary beams are all optical grade front surfaced mirrors. The same is true for the beam splitter that separates the initial laser pulse into its two components; it is only partially silvered to provide $50 \%$ transmittance and $50 \%$ reflectance. 
Initial investigations with this setup revealed several operational problems that became crucial to deal with. First, optical stability of the system became a severe problem. Highly stable optical benches were purchased for each of the mirrors used in the apparatus to replace initial elements that proved to be unsuitable for this application.

Even the introduction of these optical benches did not totally solve the problems. A CAMAC system was established io diagnose the remaining difficulties. It was determined that the lab tables at our disposal at Indiana University were not able to meet the needs of such a demanding system. Random sudden shocks would cause drastic changes in the measured values, as is illustrated in Figure 3. Here the ratio of primary to secondary signal is seen to suffer two separate drastic shifts as a result of these shocks. The answer to this problem was surprisingly simple. The entire apparatus was moved from table top to the concrete floor of the lab. After this transpositon, the effects of these random impacts on the system were almost totally eliminated.

The initial configuration of this apparatus required that the PMT be operated in a dark room, a cumbersome restriction. Also, it was seen that the results of each measurement depended slightly on the spatial placement of the PMT with respect to the two beams being measured. Here there were two distinct problems, one a logistic problem, the other a physical hardware issue. A solution to both was reached through the introduction of a signal integration module on the PMT snout. This unit is presented in Figure 4. The purpose of the integration module is twofold. First, because it is a closed system, a neutral density filter can shield the PMT from the room lights. Since the laser light is so much more intense than the room lights, a measurable signal from the laser gets through while the ambient light from the room is all but totally eradicated. The result is a spectrophotomcter that could be operated in ordinary lab lighting conditions. Once the laser beam passes through the filter, it hits a halon beam target that isotopically reflects the light into a chamber lined with more halon. All the halon has a specular reflectance of almost $98 \%$ and was made at Indiana University. This chamber is viewed by a small hole in the box that leads to the photocathode of the PMT.

This light integration chamber represents a drastic improvement in the spectrophotometer over its previous iterations. The logistic benefit of operation in lab lighting conditions is obvious. The introduction of the halon chamber produced results which were dramatic. The PMT placement was no longer of any significant importance to the measured results, since the module destroys any positional information by its very nature.

The results of these improvements were dramatic. Almost at once the spectrophotometer began to produce measurements of attenuation lengths of mineral oil to statistical levels better than expected. For a typical oil sample with an attenuation length of $28 \mathrm{~m}$ \pm 2 meters as measured by our one-meter spectrophotometer, the two-meter spectrophotometer was measuring $28.6 \mathrm{~m}$ with a statistical uncertainty of less than $.2 \mathrm{~m}$. 
The spectrophotometer has evolved over time into a system that can truly be used for quality control of mineral oils used in the MACRO experiment in Italy. Since the apparatus uses a digital oscilloscope to analyze the signals from the PMT, it requires no external support other than a pair of human hands to operate it and a stable platform or floor on which to sit. Another such system will be set up in Italy, as part of a long term plan of monitoring the mineral oils used for MACRO.

\section{Construction}

Indiana s construction responsibilities for MACRO have been the fabrication of the endplates for the vertical scintillator tanks and the construction of the calibration boats.

The scintillator tanks consist of folded-up PVC with welded endplates. The folding and welding for the vertical tanks for supermodules 1-6 was done at the University of Michigan. Indiana built these 196 endplates prior to 1991. For supermodules 7-12, which we refer to as the Attico, the vertical tank construction will be done at CalTech. We have fabricated 48 endplates for the Attico as of April 1,1991 . During the remainder of 1991 we will build the remaining 148 endplates needed to complete the Attico. We are using 0.3 FTE of a machinist for this.

A calibration boat carrying laser signals via fiber optics travels along the calibration tube in each scintillator tank. (The boat may also carry a radioactive source.) We constructed enough boats prior to 1991 that we have temporarily halted their production.

In addition to this production work, we use the machine shop for specialized projects connected with our ongoing liquid scintillator development work, such as the two-meter spectrophotometer construction discussed above and the ERP discussed below. We estimate that we use 0.2 FTE of a machinist in this way.

Thus we utilize about one half FTE of a machinist. The cost for this is provided entirely by Indiana University at no cost to DoE.

\section{E. ERP}

\section{ERP Development}

Iniana University has responsibility, along with Michigan, for the primary fast particle trigger and scintillator readout system on MACRO. This system, called the ERP (Event Reconstruction Processor), was developed by J. Musser while working at Michigan as an assistant research scientist. The trigger portion of the ERP is a look up table-driven processor which utilizes the signals observed at each end of a MACRO scintillator tank to generate a trigger based upon the amount of energy deposition in the tank. This technique is described in more detail below. This trigger provides sensitivity to all particles of interest in MACRO, including upward and downward going muons, neutrino-induced events from stellar collapse, and monopoles with $v_{m}>6 \times 10^{-4}$. 
Most important from the standpoint of stellar collapse observation, and for maximizing the acceptance of MACRO to all types of events, the trigger does not require a throughgoing particle. That is, the trigger did not require a coincidence between entry and exit faces. In addition to the trigger, high quality charge and timing readout (ADC/TDCs) of the scintillator signals is included in the ERP. The use of common circuitry for the trigger and readout electronics, when possible, results in a low cost system with a minimal number of cable interconnections. In addition to the basic capabilities necessary to trigger and read out fast particle events, additional capabilities were included specifically to maximize MACRO's capabilities with regard to the detection of gravitational collapse events. This includes a large readout buffer, necessary for dealing with the high rate of radioactive background events at the low energies characteristic of neutrino induced events from a gravitational collapse. During early 1988 an ERP system was constructed by Musser sufficient to instrument the first subsection, or supermodule, of MACRO, and was installed in the fall of 1988 . In early 1989 , a three month run on this MACRO subsection was conducted with the aim of evaluating all detector systems, as well as generating a number of physics results. The physics output from this short run on a small fraction of MACRO included a search for anisotropies in high energy cosmic rays, a study of high energy cosmic ray composition, and a search for point sources.

Based upon the performance of the system during this run, and its low overil cost, a decision was made to utilize the ERP as the primary fast particle trigger for the scintillators on MACRO, and the primary readout of the scintillator timing and energy deposition information. This decision was made in late 1985, at which time minor modifications were made to the design of the system. During 1990 the design was finalized, and production of the ERP system began with the goal of instrumenting the first six supermodules. Responsibility for this task was assumed by J. Musser, although most of the technical support for the effort remained at the University of Michigan during this period. The installation of the system on the first six supermodules is now esentially complete.

\section{ERP Future Tasks}

Money to complete the construction of the ERP system for supermodules 7-12 has now been allocated by DoE, with half the fabrication tasks to be carried out at Indiana University and half at the University of Michigan Indiana University will take overall responsibility for the task. At Indiana University, most of the techical support tasks, such as board checkout and debugging, will be handled be Mark Gephardt, a very capable technician whose salary is now being paid by Indiana University as part of J. Musser's startup package. It is expected that once ERP fabrication begins for the Attico, Mark will be devoting his full tirse to this task. This, of course, represents a valuable contribution on the part of IU towards the our DOE MACRO effort. 
The basic capabilities of the ERP system - triggering based on energy deposition in a scintillator and ADC/TDC readout of the scintillator tanks - are fully implemented at this time. However, the system design includes a number of features which have not been exploited. These features, for the most part, were included to take full advantage of MACRO as a gravitational collapse detector, and include the ability to confirm that low energy events are neutrino-induced by observing the secondary fusion $\gamma$ ray associated with charged current events in liquid scintillator. A program outlined in an OJI proposal submitted to DoE by J. Musser in the fall of 1990 , if approved, would result in a significant extension of MACRO's capabilities in the area of gravitational collapse detection through a full implementation of these features of the ERP system. The basis for this proposal, and the work planned by the group in this area, are described below.

\section{Scientific Objectives of Gravitational Collapse Upgrade}

A large class of visible supernovae (SN), known as type II supernovae, are copious neutrino emitters, occuring as a result of the gravitational collapse of the progenator star. This is brought about by the removal of degeneracy pressure in the stellar interior through inverse beta decay, a process known as neutronization. This process results in the release of about $10^{52}$ ergs of energy in the form of electron neutrinos. Subsequently, the stellar matter freefalls until the density reaches the critical value at which the equation of state 'stiffens', leading to a rebounding of the stellar material off the stiff core. The energy released during this process is roughly $10^{53}$ ergs, mostly in the form of neutrinos of all types and species. The neutrinos are expected to follow an Fermi-Dirac energy distribution with a characteristic temperature of 4-5 MeV, resulting in an average neutrino energy of about $10 \mathrm{MeV}$. The neutrino emission from a gravitational collapse event would be composed of a burst of electron neutrinos lasting less than one second, followed by the emission of all neutrino types lasting from 2 to 20 seconds. This scenario is roughly borne out by the neutrino observations of SN 1987a, although the number of events observed was too low to identify definitively a prompt electron neutrino pulse.

The generation in a gravitational collapse of a short, intense pulse of neutrinos at a tremendous distance provides an almost ideal mechanism for measuring a number of fundamental neutrino properties which are difficult to address in the lab. The most straightforward measurement is that of the neutrino mass. In the case of a non-zero neutrino mass, the difference in travel times from the supernova for two neutrinos of energy $E_{1}$ and $E_{2}$ is given by $\Delta t=(D / c) \times m_{\nu}^{2} / 2 \times\left(1 / E_{1}^{2}-1 / E_{2}^{2}\right)$. The neutrino mass limits obtained from the $\mathrm{SN} 1987 \mathrm{a}$ data, ranging from $12 \mathrm{eV}$ to $26 \mathrm{eV}$, are statistics limited. This is only partially offset by the relatively large source distance $D$, which improves the sensitivity of the measurement, and one can expect a dramatic improvement with increased neutrino flux. In this case, one can look in detail for correlations between arrival time and neutrino energy. The only source of uncertainty in this measurement stems from the model-dependent neutrino diffusion time. However, given the increased statistics associated with a gravitational 
collapse within our galaxy and improved hydrodynamic modeling of supernovae it should be possible to separate this effect from mass-dependent transit time differences.

Limits on the neutrino magnetic moment can be placed by noting that a significant change in the time profile of neutrino events occurs if neutrinos with normal helicity are flipped to the opposite helicity through electron, proton, or neutron scattering. Right-handed neutrinos (or left-handed anti-neutrinos) are unable to interact with normal matter, and therefore instantly carry a large fraction of the supernovae energy out of the neutrinosphere. In this way, limits on the neutrino magnetic moment $\mu_{\nu}$ of roughly $\mu_{\nu} / \mu_{B}<10^{-12}$ have been placed using the data from SN 1987a. This is several orders of magnitude smaller than the magnetic moment needed to solve the solar neutrino problem. However, these limits are based on the observation of only three neutrino-induced events at times greater than 2 seconds after the start of the burst in the Kamioka data, some or all of which could be background noise events, and on the rough estimate of neutrino energy emission obtained from the neutrino flux observed. It is clear that limits of this type would be on much firmer ground with higher statistics.

One of the most direct benefits derived from the observation of a stellar collapse in our galaxy would be the profound impact it would have on our understanding of the processes and conditions which lead to this class of supernovae. The vast majority of the energy emitted in a stellar collapse is in the form of neutrinos, and, therefore, the neutrino signal provides the most relevant information concerning these conditions. It would be hard to overstate the impact that a high statistics neutrino emission time profile would have on this field.

A critical question which must be addressed in evaluating the returns one can expect from further work in gravitational collapse detection is the mean time between these events in our galaxy. Unfortunately, this number is subject to large uncertainties. First, it is not at all certain that all gravitational collapse events generate significant light emission, introducing the potential for a large bias in the supernova rate based upon observed rates of visible supernovae. Second, not all supernova result from gravitational collapse. Based upon the rate of observed supernovae within our galaxy in historical times, one obtains a rate between $1 / 30 \mathrm{yr}$ and $1 / 50 \mathrm{yr}$. Studies of the number of visible supernovae remnants yield a smaller rate but are subject to very large observational biases due to the dimming with time of these remnants. The number of observable pulsars, which are thought to be the end result of a gravitational collapse, lead to rates between $1 / 30$ and $1 / 125$ years. Again, these studies are subject to large observational biases. Other estimates, based upon the number of supernovae needed to generate the heavy element abundances observed today, yield rates around 1/10 years. Finally, estimates based upon the observed rate of visible supernovae in external galaxies range from $1 / 10$ years to $1 / 50$ years, with large uncertainties introduced by uncertainties in the Hubble constant and other factors. The concensus is that the combined data when taken together point to a real supernorae rate of between $1 / 10$ and $1 / 30$ years in our galaxy. A though low, this rate is not vastly lower than the expected lifetime of the MACRO detector, and there is a reasonable chance 
that a gravitational collapse will be observed by MACRO. The scientific outcome of an observation makes the speculative nature of the search worth the risk.

\section{Characteristics of a Stellar Collapse Observation in MACRO}

Given the total energy output in neutrinos in a stellar collapse, and their energy distribution, it is straightforward to calculate the expected number of observed neutrinoinduced events in MACRO. The most important detection channel in liquid scintillator is the charged current interaction

$$
\begin{gathered}
\overline{\nu_{e}}+P \rightarrow e^{+}+N \\
N+P \rightarrow D+\gamma(2.2 \mathrm{MeV})
\end{gathered}
$$

About $95 \%$ of the signals observed in MACRO during a gravitational collapse will be due to this channel. Averaging the cross section of the primary interaction over a $4 \mathrm{MeV}$ Fermi Dirac distribution and using the observed neutrino flux from SN 1987a, one obtains an expected event count in $1 \mathrm{kton}$ of liquid scintillator of $3 \times 10^{4} / D^{2}$, where $\mathrm{D}$ is the distance of the SN from the earth in kpc. This assumes full detection efficiency. This corresponds to 300 events for a supernova at the center of our galaxy $(D=10)$, or 12 events from supernova $1987 \mathrm{a}$.

We have conducted a study of the efficiency of MACRO in detecting the positrons resulting from the charged current interaction shown above. This study includes the full effect of trigger efficiencies and positron transport losses. The result is shown in Figure 5, which contains the integral detection probability as a function of energy threshold.

The observation of a large number of low energy events in the detector occuring over a time period of seconds is the principal signature of a gravitational collapse event. However, within the characteristic energy range of the positrons produced in these events there is a significant background due to radioactivity in and around the detector, which, along with muon contamination, ultimately limits the sensitivity of a gravitational collapse search. Figure 6 indicates the typical background singles rates observed in MACRO. Based upon this background rate one can determine the sensitivity of MACRO to gravitational collapse events, knowing the expected number of events in the detector for a supernova at a given distance. Figure 7 plots the detection sensitivity as a function of energy threshold, where the sensitivity is expressed as the maximum supernova distance at a given energy threshold, requiring that the false event rate from radioactivity is less than $1 / 1000$ years. It is assumed that the search is made in two-second bins. The sensitivity plotted in Figure 7 decreases for low trigger thresholds due to the high false trigger rate from radioactivity and at high thresholds from a decrease in the positron detection efficiency. Also shown in Figure 7 is the distance corresponding to a single event observed in MACRO above a given threshold, and the sensitivity at a false detection rate of 1 per day. 
The very low rate of gravitational collapse events makes the "convincingness" of a positive observation critical. Over much of the sensitive volume implied by the result in Figure 7, the number of counts obtained from a gravitational collapse is sufficiently low as to render an uncorroborated observation unconvincing, independent of statistical arguments. What is needed is an independent means of verifying that the events in the burst are indeed neutrino-induced. Inspection of the charged current interaction above will show that such a verification procedure does exist. The neutron produced in the primary interaction is moderated in the liquid scintillator, with a characteristic moderation time of 170 microseconds. After moderation, the thermal neutron fuses with a free proton, resu'ting in a $2.2 \mathrm{MeV} \gamma$ ray. The observation of this $\gamma$ ray provides a beautiful method for cc nfirming the nature of a neutrino-induced reaction, and was, in fact, the means used by Re1.zes in the discovery of the neutrino. Their would be little doubt about the nature of a burst of low energy events, many of which were followed a short time later with a secondary event in the same tank. Conversely, a burst in which fusion $\gamma$ 's were not observed could be rejected on that basis. The ability of liquid scintillator-based detectors to take advantage of this secondary reaction represents a significant advaniage over water-based detectors, and represents tile primary focus of the proposed study.

\section{Present ERP Capabilities}

A MACRO scintillator tank consists of a 12-meter long teflon-lined PVC counter filled with mineral oil-based liquid scintillator. Each end of a tank is instrumented with photomultiplier tubes. ERP triggers are formed using a two dimensional look up table, each axis of which represents the output of the photomultiplier tube at one end of the tank. The contents of the two dimensional array contain the energy deposition that corresponds to the two inputs, and is essentially a way of correcting for the non-uniform tank response as a function of position. The present ERP system provides two trigger thresholds for each tank, corresponding to energy depositions associated with muons $(>10 \mathrm{MeV})$ and with the low energy neutrino-induced reactions from stellar collapse $(>5 \mathrm{MeV})$. The background rate above $10 \mathrm{MeV}$ is significantly lower than the muon rate, allowing the ERP to provide a clean muon trigger based on a single tank hit. This has distinct advantages over a muon trigger based upon coincident hits in an entry and exit plane. The detector acceptance is fully utilized, and is virtually independent of dead counters. In addition, this trigger allows the study of stopping particles. For low energy events, a buffer in the system supervisor stores the tank number, ADC, TDC, and timeword information for all low level triggers, and is read out when full. Studies of trigger efficiency for the low level trigger show that full efficiency is achieved at $7 \mathrm{MeV}$, falling to $50 \%$ at $5 \mathrm{MeV}$. This is significantly better than either the Kamioka or IMB detectors, and allows essentially $100 \%$ efficiency for charged current gravitational collapse events. Figure 8 shows the result of a search we made for stellar collapse during the three month spring 1989 run on the first supermodule. Plotted in this figure is the distribution of hit counts within two second bins for events above 10 $\mathrm{MeV}$, the optimal search threshold as indicated in Figure 7. 
Also shown in Figure 8 is the expected number of events, after correcting for energy threshold and time window inefficiencies, for a gravitational collapse at the center of the galaxy. This search demonstrated that there are no serious classes of non-random background, while demonstrating, on the other hand, that the complete elimination of muons from the data sample is non-trivial. In fact, muons, even ifter cuts designed to eliminate them, represent a large fraction of the events in the data sample, as determined by the energy distribution of the sample. The primary reason for this is the limited ability of the present streamer tube tracking software in handling very short muon tracks, such as occur when muons clip the corner of the detector. An extensive modification to the streamer tube tracking software needs to be undertaken to significantly reduce the muon contamination problem, work which will be undertaken as part of the proposed program.

The primary focus of our gravitational collapse program involves extending the present capabilities of the ERP system to take advantage of the potential offered by the presence of the fusion $\gamma$ signal in scintillator. A Monte Carlo study of the fusion $\gamma$ detection efficiency in MACRO has been used to demonstrate the feasibility of this program. The result of this study, which includes a full simulation of the moderation and fusion of the neutron, and the conversion of the fusion $\gamma$ in the liquid scintillator, indicates that $30 \%$ of the $\gamma$ 's deposit more than $1.5 \mathrm{MeV}$ in the scintillator tank in which the neutrino event occurs. In addition, $80 \%$ of those events deposit their energy within one meter of the position of the positron. Requiring agreement between the two signal positions reduces the background from radioactivity by an order of magnitude. Assuming a 500 microsecond window for detection of the delayed $\gamma$, an overall detection efficiency of $20 \%$ is obtained. Therefore, in order for the observation of the delayed $\gamma$ to be an effective means of confirmation of a neutrino-induced event, the chance coincidence probability within the 500 microsecond window and with position agreement within one meter, must be significantly less than 20 \%. This requires that the integral background rate above $1.5 \mathrm{MeV}$ be significantly less than $4.8 \mathrm{kHz}$ per tank. Inspection of the nominal radioactive background spectrum in MACRO, shown in Figure 6, shows that this requirement is met on average in the MACRO detector.

In order to take advantage of the presence of the fusion $\gamma$ signal, the ERP system must be made to trigger at very low energy deposition levels. The corresponding background rates make a steady state threshold at these levels impossible - the data volume would be much too large to deal with. Fortunately, the flexibility inherent in the design of the ERP makes a solution possible. Most of the logic driving the ERP trigger processor resides on programable logic arrays. By reprograming this logic, it will be possible to dynamically alter the energy threshold of the low level trigger to a value compatable with triggering on the fusion gamma signal for a specified period of time after each event. This enhancement of the capabilities of the ERP system can be accomplished by replacing a single programable logic chip in each ERP trigger module. This swap can be done after the system is installed and running, during scheduled routine maintanance periods. After this modification, the ERP system will operate in the following manner. The high level trigger will behave as before - any event satisfying the high level trigger condition will be read 
out. The low level trigger will behave as before as well, triggering on events with energy depositions greater than $5 \mathrm{MeV}$. However, for every event triggered at this or the higher level, a 500 microsecond window will open during which any event with energy depositions greater than $1 \mathrm{MeV}$ will be read out.

In addition to the modification to the trigger processor just described, a modification to the readout supervisor will be required to make optimal use of the ERP in a gravitational collapse search. The system will not be available to read out the fusion $\gamma$ event until the readout of the positron event has been completed, so the speed with which the initial readout is done is critical in determining the efficiency for detecting the fusion $\gamma$. The readout time of the system has two components - the time during which the suppression of non-participating channels is performed, and the time during which the $\mathrm{S} / \mathrm{H}$ values of participating channels are digitized. In the present system, zero suppression takes 300 nanoseconds per channel, for a total time of $\mathbf{3 0}$ microseconds per supermodule. This time can be reduced by a factor of two by a simple modification to the clock circuitry on the Supervisor module. At present, there is one Supervisor module per MACRO supermodule. The zero suppression deadtime can be reduced a further factor of two by using two Supervisor modules per supermodule, so that the fusion $\gamma$ detection inefficiency due to this deadtime can be made less than $5 \%$. The digitization time is set by the maximum digitization rate of the ADC used in the Supervisor module. The ADC used in the present circuit has a rated $1 \mathrm{Mhz}$ maximum digitization rate. In practice, however, it was found that the ADC could only operate at $0.5 \mathrm{Mhz}$ when the input contained sharp discontinuities, as is the case in this application. The corresponding event readout time for a single tank hit is 24 microseconds. To reduce this time, the ADC presently used in the Supervisor would be replaced with a more modern design - there are now many ADCs available which can digitize to 12 bits accuracy at $2 \mathrm{Mhz}$ rates. With this change, the total fusion $\gamma$ detection inefficiency due to readout deadtime can be kept below $10 \%$.

The successful use of the fusion $\gamma$ signal depends upon a number of factors, which are outlined below. Although tests performed to date indicate that readout of the fusion $\gamma$ signal is feasable, modification to the ERP system will be preceeded by a detailed study to determine the actual rejection power of this method to random bursts of radioactive background mascarading as signals from a gravitational collapse. This study will include obtaining a detailed map of the activity rates in each counter as a function of energy, particularly at very low energies. The background energy spectrum shown in Figure 6 is representative of a single MACRO counter. It is expected that there may be large local differences in activity due to differences in the surrounding materials. The successful use of the fusion $\gamma$ signal depends upon having activities in most counters at or below the levels shown in Figure 6. If areas of high activity are found in the detector, the possibility of shielding the counters in these areas will be investigated. The activity map requires that accurate calibrations be performed on each tank for low energy depositions. 
Figure 9 shows the response of a single counter to a $\mathrm{Am} / \mathrm{Be}$ neutron source, which emits neutrons with a most likely energy of $5 \mathrm{MeV}$. The energy calibration of this tank was performed by requiring the most likely energy deposition of a muon to equal $33 \mathrm{MeV}$, showing that calibrations using this method are valid at low energies. A more complete characterization of the response of the scintillator tanks to energy depositions below $5 \mathrm{MeV}$ will be part of this study. The position resolution of the scintillator tank at low signal levels is critical in determining the rejection power of this method. The best obtainable position resolution at low light levels would derive from using the relative timing between the signals seen at the two ends of the counter to determine the event position. The position resolution obtained in this way would be better than the resolution resulting from determining the event position from the relative signal amplitudes at the two tank ends, and better than the 1 meter requirement set by the energy deposition profile of the fusion $\gamma$. However, use of the TDC measurement to determine event position of very low energy events requires that the discriminator level on the timing circuitry be set much lower tinan it has been set on the ERP system in the past. There is a possibility that this would increase to an unacceptable level the sensitivity of the timing measurement to photomultiplier tube prepulsing. This would be a particular concern from the standpoint of distinguishing upward from downward-going muons. A systematic study of the effects of a lowered timing discriminator level on system performance will be undertaken as part of this program. If for this reason timing cannot be used to determine event position, the relative pulse heights at the two ends of the tank provide an additional means of acquiring the same information, but with reduced resolution.

In addition to the hardware modifications and performance study described above, software designed to monitor the gravitational collapse system in real time will be needed. This software will have two major tasks. The first task will be monitoring rates and gains for drift and making appropriate corrections in real time. At the very low thresholds needed for full sensitivity to events from gravitational collapse the rates are very sensitive to changes in gain, and it is necessary to make corrections to the calibration constants of the system and to reload trigger parameters in response to drifts to keep the data rate at manageable levels. The second task of the online software will be conducting a real time search for gravitational collapse events. It is critical that the response to an event be as rapid as possible, and this system will include the automatic notification, via electronic mail, of a list of scientists in the US and Italy in the event of a gravitational collapse candidate. An additional software task will be an extensive modification of the streamer tube tracking software to allow better rejection of muons in the gravitational collapse data sample. This will improve MACRO's sensitivity to stellar collapse by lowering the occupancy in the stellar collapse search event sample.

\section{F. Assembly}

After the pieces and parts of MACRO are fabricated and/or built, they must be assembled into a working detector. This is an enormous effort requiring dedicated contributions 
at the technician level. The vast majority of this work is done by those in residence at the Gran Sasso Laboratory (GSL). (The two-week shiftworkers contribute to an extent compatible with other goals they wish to achieve during their short stays.) Indiana University is supplying two live-in technicians (of three total for the whole US collaboration) to perform this monumental task.

As of April 1, 1991 there are six long-term (greater than two-month) Gran Sasso residents. They are three graduate students (Robert Cormack, Edward Diehl, and Lynn Miller) and three technicians (Ersilia Giusti, Larry Mossbarger, and Mike Yeakey) Three of these six residents (Miller, Giusti, and Yeakey) are from Indiana University. Boston, Michigan, and Caltech are supplying one each. In addition, Indiana University will be sending a postdoc (Scott Nutter) to the GSL on Sept. 1, 1991 to reside there for a minimum of one year.

The major 1990 DoE review of MACRO recommended that more technicians and postdocs live at the GSL. In response to that recommendation we have added Yeakey (Jan., 1991) and will add Nutter to our resident staff.

During 1991 the Indiana resident staff is working on all aspects of construction and quality control of the detector. They install and test electronics, install and fill scintillator tanks, and mount PMT's and their assemblies (reflectors, magnetic shields, bases). They find leaks in scintillator tanks and repair them.

Giusti expedites shipments between the United States and Italy, facilitating the receipt and movement of supplies and materials through customs and working with shipping companies to coordinate transportation between ports of entry and the laboratories at Gran Sasso and Frascati. She is also a bilingual assistant to all members of the American research groups. She provides translation services and helps to ease communication between American and Italian collaborators. She sees to the day-to-day needs of scientists serving their two-week shifts in Italy and of the long-term staff.

The following is a list of tasks and activities Mike Yeakey has performed for the MACRO experiment in the two-month period starting with his arrival at the Gran Sasso National Laboratory on Jan. 29, 1991.

1) For the scintillator tanks of MACRO, installation of: a) magnetic shields b) electrical leads and connectors for high voltage cables c) photomultiplier tubes and their structural supports d) miscellaneous electronics for the photomultiplier tubes

2) For MACRO monitoring, recording, and operating electronics: a) installation of Event Reconstruction Processor modules $b$ ) initialization and operation of the above equipment c) installation of CAMAC computer interface hardware d) installation and operation of LeCroy power supplies e) construction and installation of voltage correctors for tanks f) operation of control software for all the above $\mathrm{g}$ ) performing diagnostic and calibration procedures for all the above 
3) For MACRO liquid scintillator preparation: a) performed transfer of mineral oil between shipping and storage tanks at the Frascati facility b) measured and mixed scintillator (pseudocumene) and scintillator concentrate with oil c) pumped resultant liquid scintillator into experiment tanks at Gran Sasso d) followed safety procedures, and guidelines for preserving and verifying the purity of the liquid scintillator

4) General activities: $z$ ) replacing of faulty cables b) unloading of shipments of tanks and equipment (involving the operation of cranes and other industrial equipment) c) construction and modification of power cables d) performing electronic mail and database activities on VAX/VMS systems $e$ ) organizing and monitoring of tools and workspaces f) attending weekly shift report meetings $\mathrm{g}$ ) assisting graduate students and others with MACRO-related tasks

Our Indiana group is pledged to making significant contributions to MACRO at the GSL (in the trenches) where there is much work to be done to create the massive worldclass detector that MACRO will become.

\section{G. Data Analysis}

\section{MACRO as a Muon Telescope}

MACRO is rapidly becoming the wurld's premier telescope for mapping muon events from above the horizon and neutrino-induced muon events from below. Among the characteristics which make MACRO a particularly effective muon telescope are:

- Large collecting area. Since the sensitivity of a muon/neutrino telescope depends primarily on its geometric area, MACRO will be the most sensitive muon/neutrino telescope in operation when complete and will survey the sky for sources of muons/neutrinos at unprecedented flux levels. One MACRO supermodule is larger in area than Soudan 1, NUSEX, and Frejus, and comparable in size to Homestake. Currently MACRO is operating with four supermodules.

- Fine angular resolution. Studies of multimuons demonstrate that MACRO measures event positions with an angular accuracy of $\sim 1^{\circ}$. ( $87 \%$ of the events have a deviation of $\leq 1^{\circ}$.) Since the angular resolution of MACRO's streamer tube system is $\approx 0.2^{\circ}$ and the typical angular deviation of a muon detected by MACRO is approximately $1^{\circ}$, the angular error in our muon positions is dominated by the effects of multiple Coulomb scattering in rock.

- Excellent absolute measure of muon arrival time. Measurements have already demonstrated that the the short term timing accuracy is better than $1 \mu \mathrm{s}$. In addition, the event sample which is in coincidence with the EAS-Top array shows no absolute timing differences greater than $100 \mu \mathrm{s}$. The long term stability of the timing will in principle enable MACRO to search for very short periodicities like those reported for Her X-1 $(\sim 1.2 \mathrm{~s})$. 
- Continuous operation. Since MACRO continuously monitors the sky, this telescope is virtually assured of observing unexpected events like SN $1987 \mathrm{~A}$.

\section{Vertical Muon Flux}

For the results reported in this section, the data were gathered over approximately 92 days. The live time of the apparatus was 1890 hours.

Muon events include those triggers which have streamer tube tracks in which 4 or more planes were hit. To avoid reconstruction difficulties due to noisy events, those events with a large number of spurious hits were discarded unless they have the same multiplicity in both streamer tube views. Events were also excluded which have both zero zenith angle and zero azimuthal angle. If both angles were zero, then at least one view could not be reconstructed. The total number of downward events in this sample is $\approx 245,000$.

Figure 10 shows the distribution of these events in equatorial coordinates. For clarity, 1 event in every 100 is plotted. The $x$ 's on this figure are the positions of strong $\gamma$-ray emitters. Figure 11a shows the azimuthal angle distribution for these events. In Figure 11b the zenith angle distribution for these events with the GMACRO Monte Carlo superposed is shown.

A subsample of throughgoing muons was selected for further analysis. This sample includes events in which the muon trajectory crosses the apparatus from the top horizontal layer to the bottom horizontal layer of the streamer tubes. This cut selected a subsample of 96,000 muons.

In order to calculate the dependence of the vertical muon flux on depth, the sample was subdivided into bins of equal solid angle $\Delta \Omega\left(\Delta \phi=3.6^{\circ}, \Delta \cos \theta=0.01\right)$. Assuming that the zenith angle dependence of the intensity is of the form $I(h, \theta)=I_{0}(h) / \cos \theta$, the intensity at each rock depth $h$ can be written as

$$
I_{0}(h)=\left(\frac{1}{\Delta \Omega \Delta t \epsilon}\right) \frac{\sum_{i} N_{i} m_{i}}{\sum_{i} A_{i} / \cos \theta_{i}}
$$

where $N_{i}$ is the observed number of events for each of the bins at rock depth $h, m_{i}$ is the number of muons per event, $\theta_{i}$ is the zenith angle, and $A_{i}$ is the projected area of the detector. In addition, $\epsilon$ is the trigger and reconstruction efficiency and $\Delta t$ is the live time. In this equation the sums have been taken over all bins for which $h_{i}$ is within $\pm 50 / 2 \mathrm{hg} \mathrm{cm} \mathrm{cm}^{-2}$ of $h$. For this data sample the efficiency $\varepsilon$ was estimated to be $98 \%$, and independent of direction.

The observed angular distribution has been studied at each depth interval; within the errors it behaves linearly with $\sec \theta$. At our minimum depth of 3250 meters of water equivalent, the upper limit on the prompt component (deviation from $\sec \theta$ behavior) is $10 \%$. 
Figure 12 shows the vertical intensity distribution. In this figure the data include the multimuon component in order to compare our results with those of other experiments. In addition, the data have been corrected for efficiencies and rock composition. Preliminary geological surveys yield for the Gran Sasso rock an average density of $2.75 \pm 0.05 \mathrm{~g} \mathrm{~cm}^{-3}$, and rock parameters $\langle Z\rangle=9.5,<Z / A\rangle=0.499$ and $\left\langle Z^{2} / A\right\rangle=4 . \hat{6}$. Since these parameters are somewhat different from standard rock a correction was applied.

The errors shown in Figure 12 have been estimated solely from statistical fluctuations; the errors are consistent with the spread of the intensity values for different angular bins corresponding to the same rock thickness. Consequently the systematic uncertainties due to variations in rock density, or other local effects, are smaller than the statistical uncertainty. For the variations of the average density over paths of $\sim 1 \mathrm{~km}, \Delta \rho / \rho<7 \%$.

The intensity shown in Figure 12 is in reasonable agreement with previous measurements at overlapping depths within systematic and statistical uncertainties.

The data points in Figure 12 have been fit to the simple phenomenological exponential form

$$
I_{0}(h)=A e^{-h / h_{0}},
$$

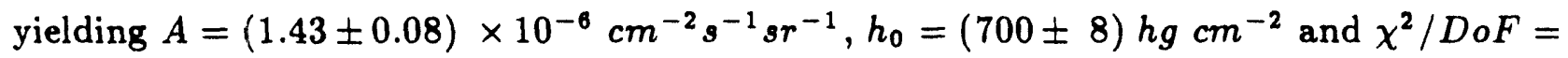
$43 / 41$. The exponential form is adequate to describe the data over the depth range shown in Figure 12.

A fit of the data to the form used by the Frejus experiment

$$
I_{0}(h)=B\left(h_{1} / h\right)^{2} \quad e^{-h / h_{1}}
$$

yields $B=(2.00 \pm 0.10) 10^{-6} \mathrm{~cm}^{-2} \mathrm{~s}^{-1} \mathrm{sr}^{-1}, h_{1}=(1134 \pm 16) h \mathrm{gcm} \mathrm{cm}^{-2}$ and $\chi^{2} / D o F=$ $61 / 41$.

The parameters of the muon spectrum, assumed to follow a power law, $d N / d E_{\mu}=$ $K E_{\mu}^{-\gamma}$ for $E_{\mu}>1 T e V$, can be extracted from the underground vertical muon intensity. Using the muon survival probability $\mathrm{P}\left(\mathrm{h}, E_{\mu}\right)$, folded with the assumed form of the spectrum, the intensity can be written as

$$
I(h)=K \int_{0}^{\infty} P\left(h, E_{\mu}\right) E_{\mu}^{-\gamma} d E_{\mu} .
$$

By replacing the survival probability curve with a step function whose value is 1 for muon energies greater than $E_{\min }=\alpha[\exp (\beta h)-1]$, we have $I_{0}(h)=K_{1}[\exp (\beta h)-1]^{-\gamma+1}$, $K_{1}=K \alpha^{1-\gamma} /(\gamma-1)$. Using for $\beta=0.4 \times 10^{-3} M W E^{-1}$, a fit to our data gives $K=$ 
$(15 \pm 3)$ and $\gamma-1=(2.73 \pm 0.03)$, with a $\chi^{2} / D o F=57 / 40$. Here MWE is meters of water equivalent.

In the above fits systematic errors have not been included. Nevertheless the parameters of these fits agree reasonably well with the parameters determined by other experiments.

In Figure 13, the muon intensity is compared with that observed by other underground experiments. MACRO clearly agrees with the results of other experiments.

\section{Small Scale Anisotropies}

A more careful analysis of the four-plane data sample shows no evidence for point sources. The data were first divided into solid angle bins $3^{\circ} \times 3^{\circ}$ wide. Background muon events were simulated by Monte Carlo methods. We first chose event positions with the observed two-dimensional distribution of zenith and azimuth angles. Arrival times were then simulated by a Poissonian process using event rates computed from the detector live-time distribution with all gaps greater than one hour taken into account. Figure 14 shows the normalized deviations from the mean. We have found there is no evidence for any excesses larger than $3.6 \sigma$. This leads to a limit on the flux from point sources of $6.6 \times 10^{-12} \mathrm{~cm}^{-2} \mathrm{~s}^{-1}(90 \%$ C.L.).

Apart from the unbiased search for point sources sketched above, a search has also been made for excess muons coming from the direction of the X-ray binaries Cyg X-3 and Her X-1.

\section{a. Cyg X-3}

We describe here the analysis of muon events collected by MACRO which point back to the region around $\mathrm{Cyg} X-3\left(P=0.19968354 d ; \dot{P}=0.904 \times 10^{-\theta}\right)$. This unusual object is a well-known periodic $\mathrm{X}$-ray binary source..$^{[3]}$ Although it has been reported as a periodic source of UHE $\gamma$ rays, ${ }^{[+]}$there has been little subsequent observational support for this claim. ${ }^{(5)}$ The extreme variability of $\mathrm{Cyg} X-3$ at all wavelengths may account for this behavior. In the past there have also been two positive observations of an underground muon signal from the direction of $\mathrm{Cyg}$ X-3 modulated with the 4.8 hour X-ray period. ${ }^{[(]}$ Other underground experiments, however, have failed to detect a signal. ${ }^{[7]}$ The situation regarding underground muons from $\mathrm{Cyg} \mathrm{X}-3$ is at present uncertain primarily because this source is highly variable and the measurements are usually neither contemporaneous nor at the same depth. 
The muon data were collected by the first MACRO supermodule (12 $m \times 12 m \times$ $5 \mathrm{~m}$ ) during two extended data runs. The spring 1989 data (sample 1) were obtained from February 27 to May 30, 1989 with a total detector live-time of $1704.8 \mathrm{hr}$; the fall 1989/spring 1990 data (sample 2) were obtained from November 14, 1989 to May 10, 1990 with a total detector live-time of $3021.3 \mathrm{hr}$.

The data analyzed have been selected by somewhat different criteria than used in our MACRO paper based on the spring 1989 data.$^{[2]}$ In the present study, muons with successfully reconstructed tracks were retained if they crossed at least 4 out of 10 streamer tube planes, and had zenith angles $\theta \leq 80^{\circ}$. For isotropic events satisfying these criteria, the first MACRO supermodule had an acceptance of $S \Omega \sim 500 \mathrm{~m}^{2} \mathrm{sr}$. Our preliminary estimate of the angular resolution is $3^{\circ}$. In addition, muon events in this study were required to have equal multiplicities in both projected views of the streamer tubes, and to have come from runs of one hour or longer in duration. In the fall $89 /$ spring 90 data, certain technical cuts were also required to eliminate poorly reconstructed tracks which resulted from minor problems with the electronics. There were 207,045 events in sample 1 and 328,745 events in sample 2 which satisfied our selection criteria.

The data used in the search for muon signals from Cyg X-3 included the events in samples 1 and 2 with tracks pointing back to a $10^{\circ} \times 10^{\circ}$ window centered on the 1989.5 coordinates of Cyg X-3. This window agrees in size with the windows used in the analyses by Soudan and NUSEX. ${ }^{[\theta]}$ The numbers of events, $n$, we find in this window are listed in Table 1.

Table 1. Analysis of the MACRO Cyg X-3 Muon Data

\begin{tabular}{|c|c|c|c|}
\hline & Spring 1989 & Fall 1989/Spring 1990 & Total \\
\hline $\mathrm{n}$ & 639 & 1,001 & 1,640 \\
\hline $\mathrm{n}_{6 k d}$ & 631 & 982 & 1,613 \\
\hline \multicolumn{2}{|c|}{$F_{\text {otdy }}\left(\mathrm{cm}^{-2} \mathrm{~s}^{-1}\right)^{\dagger}<1.2 \times 10^{-11}$} & $<9.8 \times 10^{-12}$ & $<8.3 \times 10^{-12}$ \\
\hline$n \bar{R}^{2}$ & $8.6 \times 10^{-3}$ & 0.70 & 0.71 \\
\hline $\mathrm{P}\left(>n \bar{R}^{2}\right)$ & 0.96 & 0.50 & 0.88 \\
\hline$\Upsilon_{n}$ & 11.57 & 12.42 & 13.36 \\
\hline$P\left(>\Upsilon_{n}\right)$ & 0.31 & 0.37 & 0.38 \\
\hline$F_{\bmod }\left(\mathrm{cm}^{-2} s^{-1}\right)$ & $<6.7 \times 10^{-12}$ & $<4.9 \times 10^{-12}$ & $<4.6 \times 10^{-12}$ \\
\hline
\end{tabular}

$+90 \%$ C.L. 
Background muon events were simulated individually for samples 1 and 2 by Monte Carlo methods. We first chose event positions with the observed two-dimensional disiribution of zenith and azimuth angles. Arrival times were then simulated by a Poissonian process using event rates computed from the detector live-time distribution with all gaps greater than one hour taken into account. The times for events in a $10^{\circ} \times 10^{\circ}$ window about the position of $\mathrm{Cyg} X-3$ were recorded and analyzed in the same way as the data events. More than $3 \times 10^{6}$ muon events in the Cyg X-3 window were simulated in this manner. The average number of background events for each data set, $\mathrm{n}_{b k d}$, is given in Table 1. Table 1 shows that there is no signal from the region around Cyg X-3 at the $1 \sigma$ level.

The upper limit to the steady muon flux at the $90 \%$ C.L. was calculated by $(n-$ $\left.n_{\text {bkd }}+1.28 \sqrt{n}\right) /\left(\epsilon f A_{\text {eff }} t\right)$, where $\epsilon=0.65$ includes both detection and track reconstruction efficiencies, $f=0.66$ is the fractional time Cyg X-3 is at least $10^{\circ}$ above the horizon, $A_{\text {eff }}=130 \mathrm{~m}^{2}$ is the effective area of MACRO for the Cyg X-3 window, and $t$ is the live-time. The efficiency quoted above is based upon our very conservative estimates of pointing and reconstruction errors. We expect the efficiency to be significantly improved in the near future. The upper limits, $F_{\text {stdy }}$, for the three data sets are given in Table 1.

In the search for periodic muon signals from Cyg X-3, a correction was applied to the arrival time of each event to account for the earth's motion about the solar system barycenter. ${ }^{(8)}$ Sample 1, sample 2, and the complete data set were searched separately for a muon signal modulated by the 4.8 hour X-ray period using the parabolic ephemeris of van der Klis and Bonnet-Bidaud. ${ }^{[3]}$ The phase diagram for the complete data set is shown in Figure 15 as a solid line and the background is shown as a dashed line. This figure shows no signal greater than $2 \sigma$ above background. There is similarly no signal greater than $2 \sigma$ above background in the phase histograms for samples 1 and 2 .

We then searched for evidence of a statistically significant muon signal from the $10^{\circ} \times$ $10^{\circ}$ window around Cyg X-3 modulated by the 4.8 hour X-ray period ${ }^{(3)}$ using both the Rayleigh test and the Protheroe test. In Table 1 we list the Rayleigh power, $n \bar{R}^{2}$, for the three data samples. ${ }^{[\theta]}$ Both samples 1 and 2 were considered as coherent data samples in the computation of the Rayleigh power. For the total data sample, the Rayleigh powers were added incoherently. In Table 1 we also give the probability, $\mathrm{P}\left(>n \bar{R}^{2}\right)$, that the Rayleigh power has a value at least as large as we found for events distributed uniformly in phase (a test of the null hypothesis). This probability was computed using the simulated Monte Carlo events to account properly for the gaps in the live-time distribution. As shown in this table, the Rayleigh test implies that the muon events in the $10^{\circ} \times 10^{\circ}$ window are not modulated by the 4.8 hour X-ray period.

We have made a similar investigation using the Protheroe test. ${ }^{[10]}$ Our computation of the Protheroe power $\Upsilon_{n}$ is given in Table 1 along with the probability $P\left(>\Upsilon_{n}\right)$ that the Protheroe power has a value at least as large as we found for events distributed uniformly in phase. This probability has also been calculated using the simulated event distributions. 
The Protheroe test also implies that the events in a $10^{\circ} \times 10^{\circ}$ window around $\mathrm{Cyg} \mathrm{X}-3$ are not modulated by the 4.8 hour X-ray period. In the last row of Table 1 we estimate the upper limit, $F_{\text {mod }}$, to the modulated flux from $\mathrm{Cyg}_{\mathrm{g}} \mathrm{X}-3$, computed for the phase bin with the largest excess above background. In Figure 16 we show the MACRO upper limit compared with the results of other underground experiments. (8,7,11] $^{-1}$

\section{b. Her X-1}

Her X-1 is also a periodic source of $\mathrm{X}$ - to UHE $\gamma$ rays. It is a close $(\approx 6 \mathrm{kpc})$ eclipsing binary system containing a neutron star and a low-mass companion star $\mathrm{HZ}$ Herculis. In the $\mathrm{X}$-ray, several periods have been reported: $P=1.2378 s, P=1.7 d$, and $P=35 d$. For Her X-1 there has been no underground muon signal reported. A recent EAS observation, however, has found an anomalous muon content in air showers. ${ }^{[12]}$ The period found from this measurement, $P=1.2335 \mathrm{~s}$, differs significantly from the $\mathrm{X}$-ray period.

As for Cyg X-3, events in a $10^{\circ} \times 10^{\circ}$ window around Her X-1 have been searched for a statistically significant signal modulated by the pulsar and orbital periods. The analysis shows no evidence for a modulated signal from Her X-1. For the modulated signal in the $10^{\circ} \times 10^{\circ}$ window, the bin in the phase plot with the largest excess yields a flux limit of $1.3 \times 10^{-12} \mathrm{~cm}^{-2} \mathrm{~s}^{-1}(90 \%$ C.L. $)$.

\section{c. $\quad 1 \mathbf{E} 2258+586$}

The X-ray pulsar $1 \mathrm{E} 2259+586$ is located at the center of G109.1-1.0, a supernova remnant. Searches have been made for $\mathrm{TeV}$ and $\mathrm{PeV} \gamma$-rays. No evidence has been found so far. ${ }^{[13]}$ In addition, there have been searches for muons in underground detectors. There is weak evidence for a muon flux has been reported by Soudan 1; a modulation was observed with a period of $6.979 \mathrm{~s}^{[1+]}$

Events in a $10^{\circ} \times 10^{\circ}$ window around $1 \mathrm{E} 2259+586$ have been searched for a statistically significant signal modulated by the pulsar $(3.49 \mathrm{sec})$ and orbital $(38.30 \mathrm{~min})$ periods. The analysis shows no evidence for a modulated signal from Her X-1. For the modulated signal in the $10^{\circ} \times 10^{\circ}$ window, the bin in the phase plot with the largest excess yields a flux limit of $1.3 \times 10^{-12} \mathrm{~cm}^{-2} \mathrm{~s}^{-1}(90 \%$ C.L. $)$.

\section{WIMPS from the Sun}

A number of different lines of evidence have suggested that most of the matter in the universe is nonluminous. ${ }^{[15]}$ Cosmological studies of deuterium, ${ }^{3} \mathrm{He}$, and ${ }^{4} \mathrm{He}$ imply that this dark matter is nonbaryonic. ${ }^{[16]}$ One possibility for the identity of this dark matter are a class of particles collectively known as WIMPS (Weakly Interacting Massive Particles) for their weak interaction cross section and their mass which is orders of magnitude greater 
than a proton. ${ }^{[16]}$ If they exist, it is thought that these particles were created in the Big Bang. The WIMPS would then be gravitationally concentrated in the galaxy and many would eventually be captured by the sun or the earth. The annihilation of these WIMPS would result in an observable $\nu_{\mu}$ signal from the sun or earth if there were enough Dark Matter created in the Big Bang to provide the missing mass required by the dark halo in our Galaxy, $\sim 0.4 \mathrm{GeV} / \mathrm{cm}^{3}$.

We have analyzed the data from the spring 1989 and fall 1989 data runs to determine upper limits to the WIMP signal from the sun. These limits were based on Monte Carlo calculations of the signal expected for annihilations of the SUSY particles photinos and higgsinos. ${ }^{[17]}$ In Figure 17 we have used the $90 \%$ C.L. on the neutrino flux from the sun to compute a limit on the density of dark matter in our Galactic halo. In these computations we have assumed that there is a steady state between the number of annihilations and the number of captures of the WIMPS. At the limit of $\sim 0.4 \mathrm{GeV} / \mathrm{cm}^{3}$, the WIMP candidate would provide the dark matter in the Galactic halo.

\section{Moon Shadowing}

We would like to look for a decrease in the number of primary protons coming from the direstion of the Moon (Moon shadowing). This would establish the absolute pointing accuracy of MACRO.

However, protons travelling to the earth past the moon are deflected by the magnetic field due to the earth's magnetic dipole moment. To estimate this deflection, it is assumed that the proton's zenith angle is $90^{\circ}$ for simplicity, and the magnetic field is 0.3 gauss at the earth's surface and 1.4 microgauss at the moon's surface. A proton of momentum $\mathrm{p}=1.64 \mathrm{TeV} / \mathrm{c}$ bends by an angle $\theta \approx 1^{\circ}$ as it travels from the moon to the earth (with $p_{\theta}$ constant).

The muon threshold for reaching a distance $l$ KMWE under the earth is

$$
E_{t h}(T e V)=0.53 \times[\exp (+0.4 l)-1],
$$

or $1.23 \mathrm{TeV}$ for $l=3 \mathrm{KMWE}$. For a muon of this energy, the primary proton energy must be higher, usually much higher. For example, the average number of muons at $3 \mathrm{kmwe}$ coming from a $1.64 \mathrm{TeV}$ primary proton is only $3.7 \times 10^{-6}$. Since the typical energy of a primary proton which produces a muon seen by MACRO is in excess of $5 \mathrm{TeV}$, the deflection of the primary proto . 'i the earth's field should be negligible with respect to MACRO's angular resolution. Therefore, there may be a measurable decrease in the number of muons seen at the position of the moon.

Our analysis shows no evidence for moon shadowing, although it is likely that not enough events have yet been collected to see the effect. 


\section{MACRO Monte Carlo}

We have developed a single atmospheric muon generator for the GMACRO Monte Carlo. This generator is based upon the intensity distribution derived above.

In addition, we have propagated the observed distribution of muons at the surface of the mountain to Hall B of the Gran Sasso Laboratory using the known rock overburden. We have used the CERN routine GEANT to propagate the neutrinos through the rock. We now believe we understand the distribution of single muons observed by MACRO.

\section{H. Air Cerenkov Detector Development}

Our group, along with the Astrophysics Group at the University of Michigan, has embarked on a development project whose goal is the deployment of an Air-Cerenkov telescope array on the Gran Sasso mountain. The scientific objectives of the project are two-fold. First this array will verify that the muon trajectories determined by the track finding algorithms correctly point back to the sky within the angular accuracy $\left(\approx 1^{\circ}\right)$ of MACRO. Using MACRO alone, there is no method of verifying that the muons seen are actually coming from the reconstructed direction. (Doing this by observing the shadow of the Moon, as discussed above, is difficult because of the large data sample which is needed.) It is absolutely essential in conducting point source searches that the reconstructed direction be correct, given the very low signal rate expected. An Air-Cerenkov detector deployed on the top of the Gran Sasso can be surveyed very accurately, and its pointing direction cross-checked by aligning it with stars at known positions. We plan to set the angular acceptance of the Cerenkov detectors by stopping down the aperture of each telescope to the solid angle subtended by MACRO on the sky. The telescope will then be oriented so that it points back to MACRO. Offline showers will be analyzed if there is a coincidence with a muon event seen by MACRO. The coincidence rate for a five detector array is such that a pointing verification measurement is possible in a relatively short (few month) period of run time.

The second scientific objective of the air Cerenkov-MACRO combination is a measurement of the energy dependence of the Cosmic Ray composition at energies above 2 $\mathrm{TeV}$. The composition is determined using the muon multiplicities observed by MACRO and the primary particle energy determined by the Air-Cerenkov array. This technique is now being used with the MACRO/EASTOP combination at higher energies [1]

Our group is playing a leading role in the simulation of this experiment using Monte Carlo routines incorporating the best available cosmic ray shower code, with the goal of examining in detail the feasibility of this technique. We are also working with the University of Michigan in conducting a series of tests of a five telescope prototype array. Our group has supplied a data acquisition system and readout electronics for this test, and will participate in the data analysis. 


\section{III. $\mathbf{L}^{\star}$}

\section{A. Status}

The status of $\mathrm{L}^{\star}$ can be summarized most succinctly by the following two messages. The first is excerpted from the SSC Program Advisory Committee (PAC). The second is a memo from SSC Director Roy Schwitters presenting his actions after receiving the PAC report.

\section{Excerpt from SSC PAC}

Report of the March 10-12, 1991 Meeting of the SSC Program Advisory Committee

The PAC recommends that $L^{\star}$ be supported to proceed toward the development of a technical design report, subject to the prompt development of scientific and technical leadership that is acceptable to the SSCL Director. The Committee urges the laboratory to act in concert with the collaboration to accomplish this objective.

The PAC notes that the SSCL expects to contribute a total of $\$ 550$ million (FY 90 dollars) to two major detectors, and will use this funding flexibly, based on scientific merit and need. The probable range is $\$ 225 \mathrm{M}$ to $\$ 275 \mathrm{M}$ for each detector.

\section{Memo from Roy Schwitters}

From Roy F. Schwitters, March 20, 1991, Decision Memorandum II - on aspects of the initial scientific program for the SSC.

Based in part on advice received at the March 10-12, 1991 meeting of the SSCL Program Advisory Committee (report attached) and on other recent developments, the following decisions pertaining to the scientific program of the Laboratory are hereby announced:

1) The Laboratory seeks a successful $L^{\star}$ technical proposal/design report by the April 1, 1992 deadline that will lead to a scientific program fully competitive with that expected for SDC. We view positively the recent actions aimed at broadening the technical and scientific leadership of $L^{\star}$. This indicates to us a willingness on the part of $L^{\star}$ to resolve with the Laboratory the important collaboration and management issues that remain. The Laboratory will hold intensive discussions in the very near future with the proposed leadership of $L^{\star}$, including US and foreign participants, to resolve questions of scientific and technical leadership, management structure, participation by US groups, involvement of international partners in the scientific programs of the Laboratory, and the SSC Laboratory's role in detector construction and operations. 
2) The Laboratory agrees with the PAC's assessment that the combination of an $L^{\text {* }}$ detector with the SDC can provide an outstanding initial scientific program for the SSC, with significant complementarity. This excellent program will not be possible without the full intellectual and material participation of the international community.

3) Over the next six months, the Laboratory will develop with the leadership of SDC a joint Laboratory-collaboration management structure for its construction and operation.

4) The very large scale of the detectors proposed by SDC and $L^{\star}$, the fixed cap for US/DOE funding for the initial phase of SSC detectors, and current uncertainty concerning foreign contribution to these detectors indicate the following guidance to SDC and $\mathrm{L}^{*}$ in the preparation of technical proposals/design reports:

a) The general physics goals and priorities as outlined in the LOI's shouid be followed, recognizing that some reduction in scope and staging will likely be required to meet cost const raints.

b) Detector management must develop a design-to-cost approach.

c) A US/DOE funding base of $\$ 225 \mathrm{M}$ should be assumed for the initial phase of each detector. As much as $\$ 275 \mathrm{M}$ may be allocated to a detector at the outset pending PAC and Laboratory review. The remainder of the $\$ 550 \mathrm{M}$ set aside for the two large detectors will be allocated over the course of detector construction. The intent in reserving these funds will be to maintain flexibility to optimize the overall scientific program and to provide equal scientific opportunity for both SDC and $L^{\star}$.

d) Each technical proposal/design report must make a convincing case to the Laboratory and review groups that its total cost will not exceed $\$ 500 \mathrm{M}$ (US accounting methods) unless firm foreign commitments exist that enable the detector budget to exceed this amount.

\section{B. Role of Indiana Group in $\mathbf{L}^{\star}$}

Our group is part of the Central Tracker Group, which will develop the high-resolution, fully hybridized central tracker to be used by $L^{\star}$ at the SSC. The full tracker would use silicon strip detectors, scintillating fibers, and straw drift tubes in a magnetic field. Its primary function is to reduce backgrounds from conventional QCD processes in order to facilitate the search for new phenomena at the SSC. Our efforts will be concentrated on the evaluation of technical details associated with the straw tube detectors, including the optimization of detectors and materials for use in the high rate and high radiation SSC environment in the small $L^{\star}$ central cavity. Work at Indiana University will be concerned with Monte Carlo studies of drift tube array performance, optimization of the drift chamber gas, and the development of a suitable readout system for the drift tube system. 


\section{Drift Tube Gas}

As part of the development of the drift tubes for the central tracking system for the SSC, Indiana University has participated with Boston University in the development of a system to locate an optimum drift tube gas for the SSC environment. IU has concentrated its efforts on the optimization of the drift velocity of this gas. These efforts require the use of a gas analysis system capable of maintaining high levels of sample purity. Using a drift chamber constructed at IU early in 1991 as the heart of this system, a gas network has been designed that should meet all the operational specifications necessary for such a detailed study. Initial measurments of drift velocities with this drift chamber using a low quality gas network have shown that it is quite capable of making the measurments demanded of it.

The gas analysis for the central tracker will not actually be done with the straw tubes themselves. Since the electric field of a cylindrical tube is radially dependant, the drifting electrons would have an acceleration towards the wire that would make comparative measurment of the drift velocities difficult due to the nonuniform electric field. It is clear that in order to measure the drift velocity as a function of applied electric field, it is desireable to have a homogeneous electric field. Such an apparatus would not resemble a tube as such, but rather a box with field shaping wires to provide the homogeneous properties the electric field would require. At the end of this electric field region, sense wires would collect the drift electrons, which have been amplified to some measurable level.

During the past year, parts for a pair of such drift chambers were constructed at Indiana University. These prototypes, shown in schematic in Figure 18, have an active drift region $5 \times 2.5$ inches in size, although the eleciric field is currently thought to be homogeneous over a somewhat smaller region. The cathode is separated from seven sense wires by 16 field shaping wires that form the homogenous electric field necessary. At the end of this series of field shaping elements are the seven sense wires between two fine meshes intended to provide amplification for the final signal.

Though parts for two such chambers have been produced at Indiana University, only one chamber has currently been constructed. This unit currently resides at Indiana University where the drift velocity component of the studies are being carried out. The other chamber, still to be assembled, will reside at Bosion University where the magnetic field properties of the gases will be evaluated.

Actual operation of the functional chamber has indicated that it will be able to establish the drift velocities of each gas for a wide range of applied electric fields. In trial runs using argon-ethane in a 50:50 mixture as a standard gas, the drift velocity curve shown in Figure 19 was measured. This result matches well the anticipated curve for argon-ethane. These data were taken using a far from optimum gas system that used oil bubblers for pressure regulation and no temperature regulation. Efforts are needed to extend the quality of the gas system and environmental monitoring that will improve the operation of this chamber. 
The design of the high purity gas system demands that several major concerns be addressed. A schematic of the final design is presented in Figure 20. First, any component of the gas network must be made of a material that is immune to chemical degradation over extended exposures. Also, to insure purity of the gas sample, these same materials must be non-permeable to atmospheric contaminants such as carbon dioxide, nitrogen, oxygen, and water vapor. From the list of availiable materials, Teflon PFA (perfluoroalkoxy) was selected as the best since it shows characteristics of chemical inertness and non-permeability that are unsurpassed. Investigations into the availability of gas system components have shown that almost every form of tubing, valve, and fitting are availiable in PFA.

Inquiries into commercial systems currently availiable to provide custom gas mixtures to high tolerances proved encouraging. These controllers typically drive mass transducers which manage the flow of each of the constituan $t$ gases. After careful research into the commercial alternatives, it is certain that a gas mixing system can be purchased that will fit the needs of these studies.

After each gas is run through the drift chamber, it is important that the entire system be thoroughly cleaned out before the next mixture is tested. First, the entire system should be emptied to vacuum with a pump. This not only clears out old gas from the chamber and its gas lines, but over time also removes heavy contaminants (such as water) that might have worked their way into the system. After this, gas of the desired composition should be allowed to flow through the system until a sufficent purge is achieved.

Since the system to analyze the gases is not a straw tube array, it is not subject to the structual limitations of straws. The process of pumping out straw tubes would cause them to collapse under the vacuum. The drift chamber can trivially be pumped down to zero pressure with no problems since it is constructed totally of rigid components. The gas system itself is also very rigid. The most flexable element of the entire gas flow network will be the PFA tubing used to flow the gas, and this is rated to over $10^{6} \mathrm{~Pa}$ (almost an order of magnitude over the $10^{5} \mathrm{~Pa}$ that 1 atmosphere of pressure caused by a vacuum pump would produce.)

As part of the quality control process for the experiment, an independent analysis of the constituants of the gas (as well as contaminant levels) is necessary. Inquiries were made at several labs through the U.S. which provide high accuracy gas chromotragraphy services. The information they can provide generally falls into two catagories. First, the ratio of the major components of the gas in question can be determined. This analysis can be compared to the ratios that the gas mixing system was set to produce. Any difference outside the operating specification can be noted and corrected. The second piece of information involves the level of contamination of the gas. Contaminant searches typically include such compounds as carbon dioxide, oxygen, nitrogen, and water. If an uncomfortable level of any of these pollutants is indicated, steps must be taken to remove it from the gas flow system. 
In summary, as part of our efforts in 1991, we constructed parts for two identical drift chambers. One of these chambers, not yet assembled, will be used at Boston University to test the magnetic field properties of the SSC gases. The other chamber was tested, and has been found to be operational for the evaluation of drift velocities in a constant electric field. In order to facilitate these measurements at Indiana University, an entire gas flow network has been designed which has stringent quality control methods that will insure high quality measurements.

\section{Drift Tube Readout}

Work on the drift tube readout system for $L^{\star}$ in 1991 will center on developing fully hybridized front end amplification electronics which can tolerate the high radiation environment at the SSC, and which minimize the number of radiation lengths represented by the front end electronics. Also critical is the development of techniques for getting the drift tube signals out of the central tracking region without introducing large dead regions in the detector acceptance. In this regard, we will investigate the use of fiber optic interconnections between the front end electronics and the remote readout electronics, as well as signal multiplexing schemes. 


\section{REFERENCES}

1. The MACRO Collaboration, Physical Review D 42, 1396 (1990)

2. The MACRO Collaboration, Phys.Lett.B 249, 149 (1990)

3. M. van der Klis, and J.M. Bonnet-Bidaud, Astron.Ap., 214, 203 (1989)

4. M. Samorski and W. Stamm, Ap.J., 268, L17 (1983).

5. D.J. Fegan, 21st International Cosmic Ray Conference, Adelaide, Australia. 11, p. 23.

6. G. Battistoni et al., Phys.Lett. 155B, 465 (1985); M. Marshak et al., Phys.Rev.Lett. 54, 2079 (1985).

7. Y. Oyama et al. Phys.Rev.Lett., 56, 991 (1986); Ch. Berger et al. Phys.Lett.,174B, 118 (1986); R. Bionta et al. Phys.Rev.Lett., 36, 30 (1987); Corbato, S., University of Pennsylvania, unpublished Ph.D. dissertation (1989).

8. Space Telescope Data Analysis System (STSDAS), Space Telescope Science Institute, Baltimore, MD

9. O.C. de Jager, Potchefstroom University, unpublished Ph.D. dissertation (1987).

10. R.J. Protheroe, Astronomy Express, 1:4-6, 137 (1985)

11. M. Aglietta, et al 1990, 21st International Cosmic Ray Conference, Adelaide, Australia. 9, p. 388; S. Corbato, et al 1990, 21 st International Cosmic Ray Conference, Adelaide, Australia. 8, p. 398; K. Johns, et al 1990, 21 st Internctional Cosmic Ray Conference, Adelaide, Australia. 9, p. 402; L.S. Peak, 1990, 21 st International Cosmic Ray Conference, Adelaide, Australia. 11, p. 332

12. Dingus, B.L. et al. Phys.Rev.Lett., 61, 1906 (1988)

13. Cawley, M.F. et al. 1987, 20th International Cosmic Ray Conference, Moscow, U.S.S.R. 1, p. 240; Bloomer, S.D. et al. 1987, 20th International Cosmic Ray Conference, Moscow, U.S.S.R. 1, p. 248.

14. Ruddick, R. 1987. in Very High Energy Astronomy, ed. K.E. Turver (Dordrecht: Reidel), p. 185

15. Binney, J., and Tremaine, S. 1987 Galactic Dynamics, (Princeton, NJ: Princeton University Press), chap. 10.

16. Kolb, E.W., and Turner, M.S. 1990 The Early Universe, (Redwood City, CA: Addison-Wesley)

17. Ritz, S., and Seckel, D. Nuclear Physics, B304, 877 (1988). 


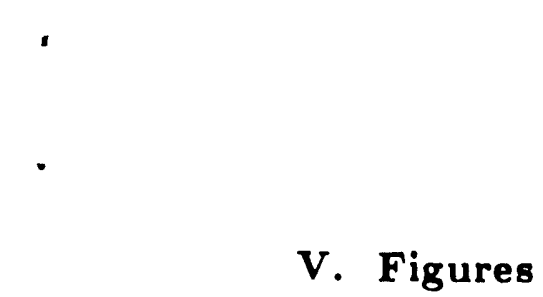




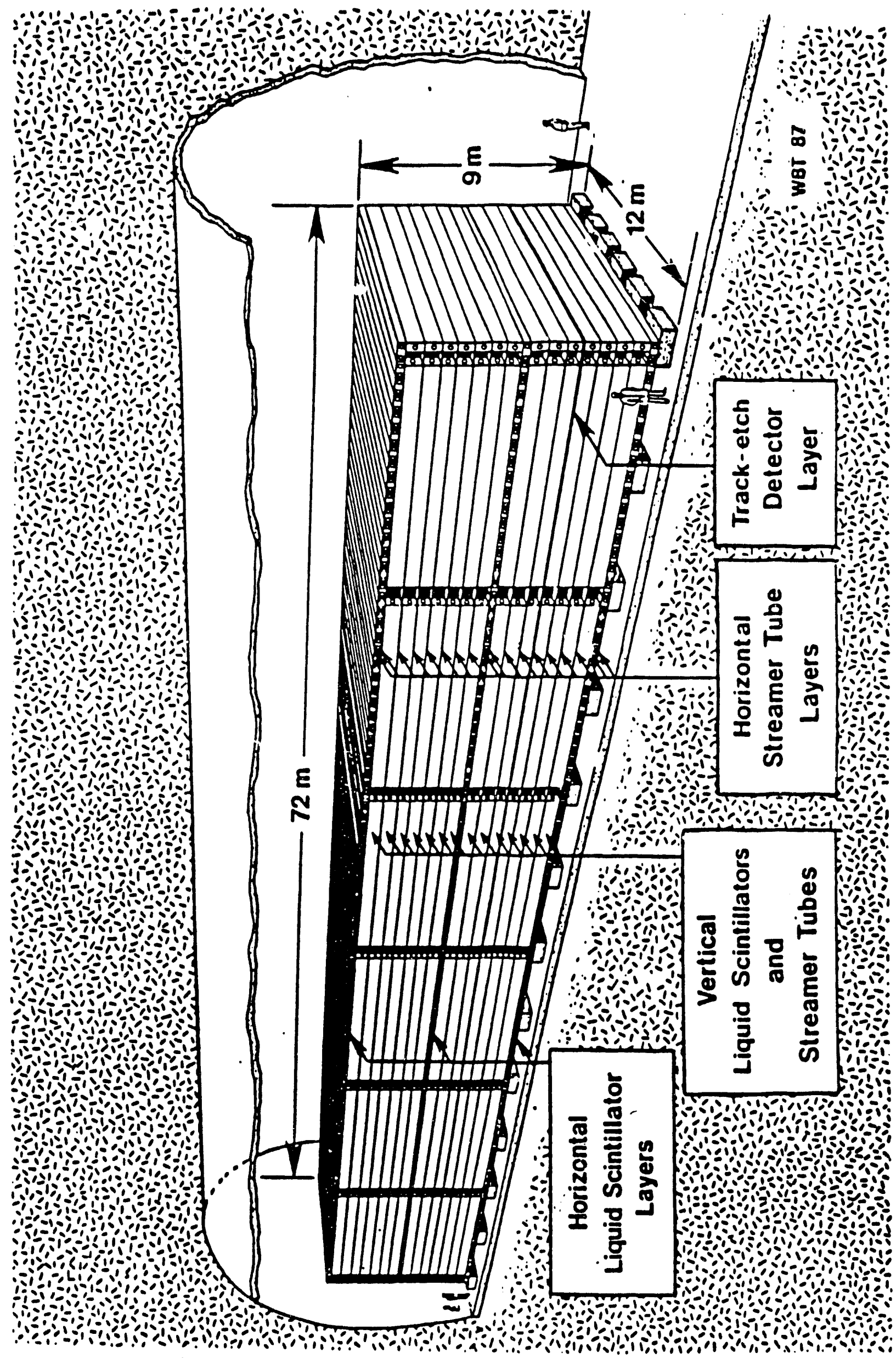

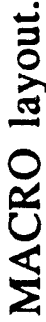

$\frac{5}{5}$ 


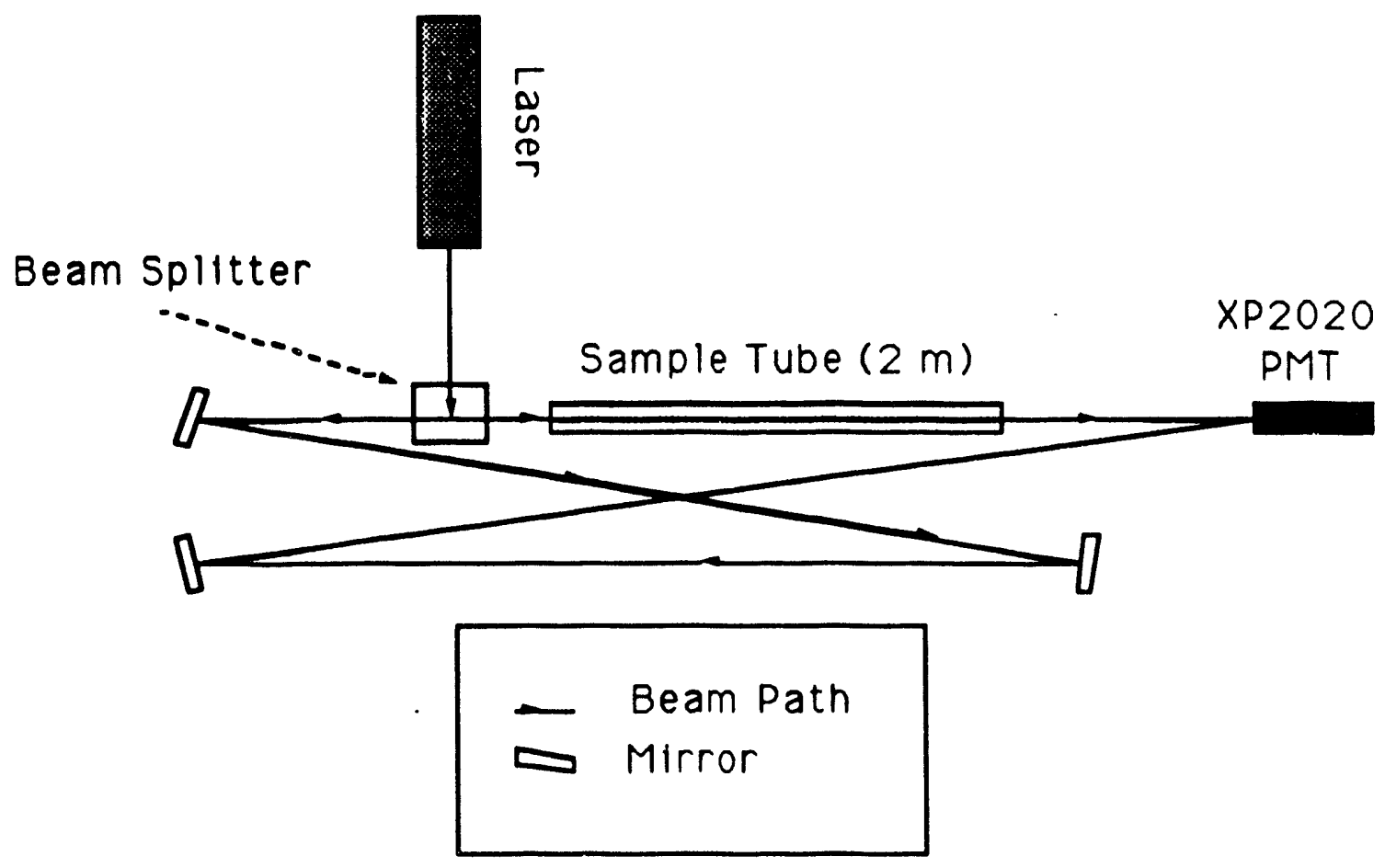

Figure 2. Schematic drawing of two-meter spectrophotometer. 
Effect of Random Shock on Ratio

Target Out

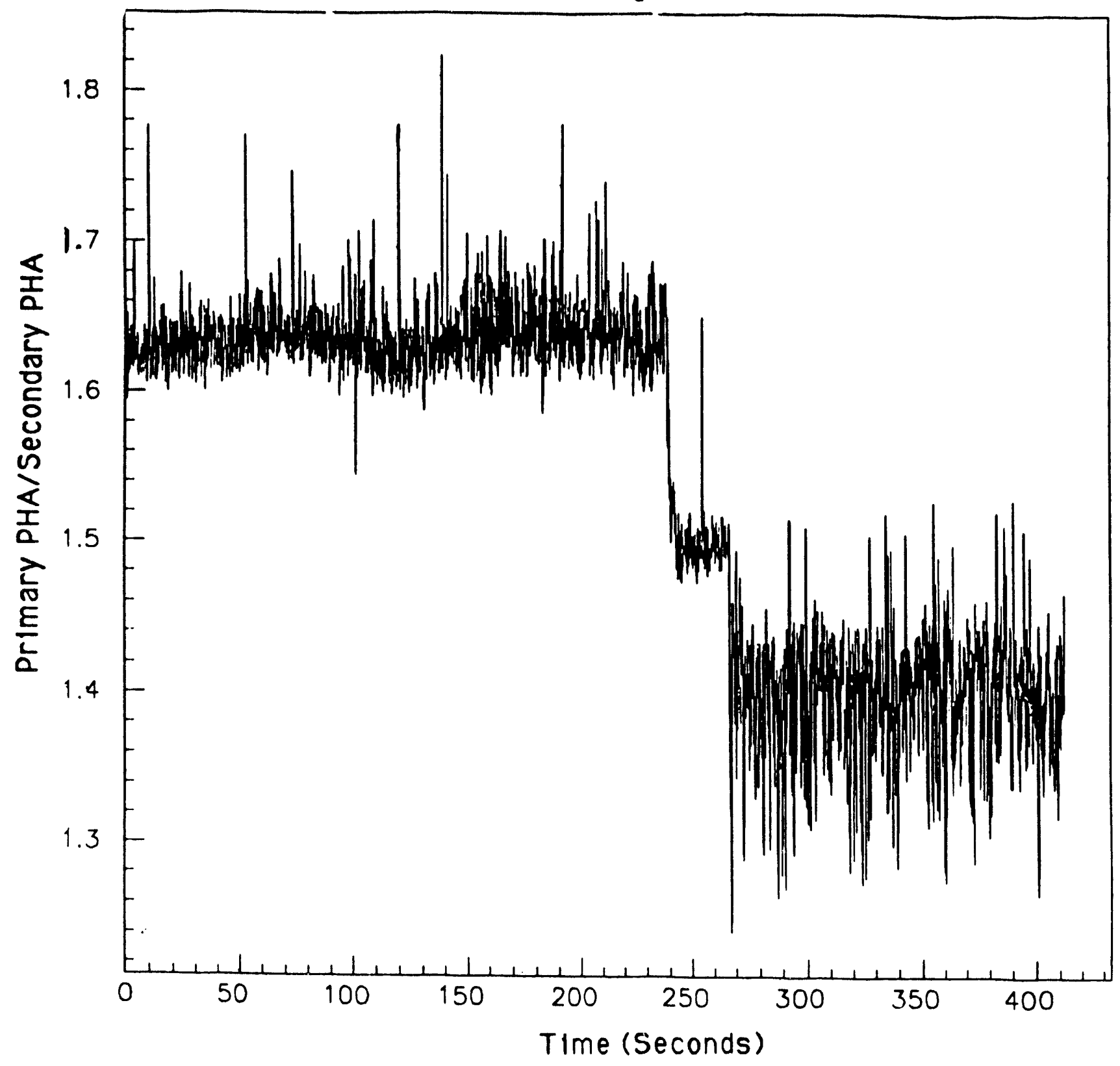

Figure 3. Ratio of primary to secondary pulses as a function of time. 

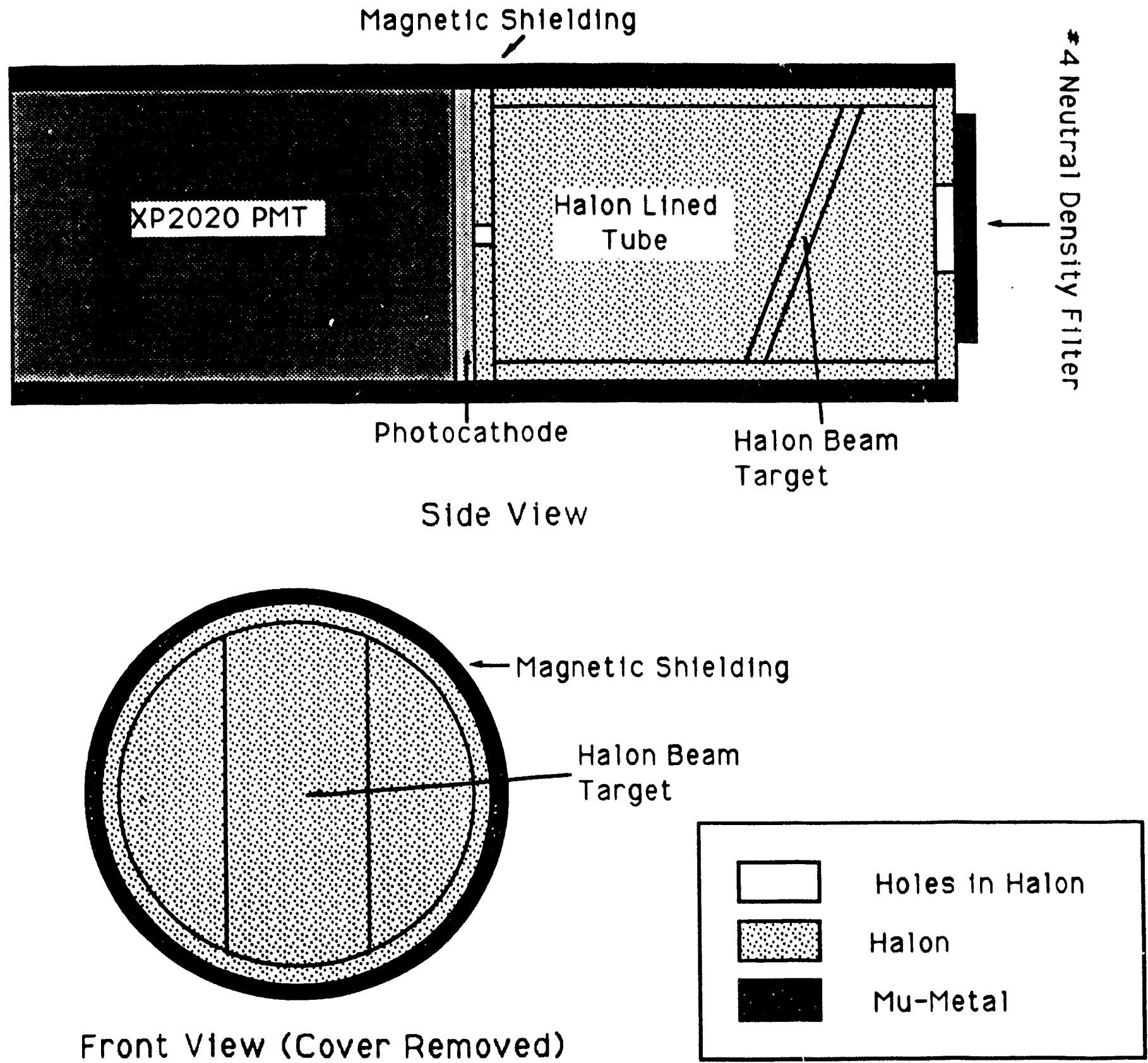

Figure 4. Phototube for two-meter spectrophotometer with signal integration snout. 


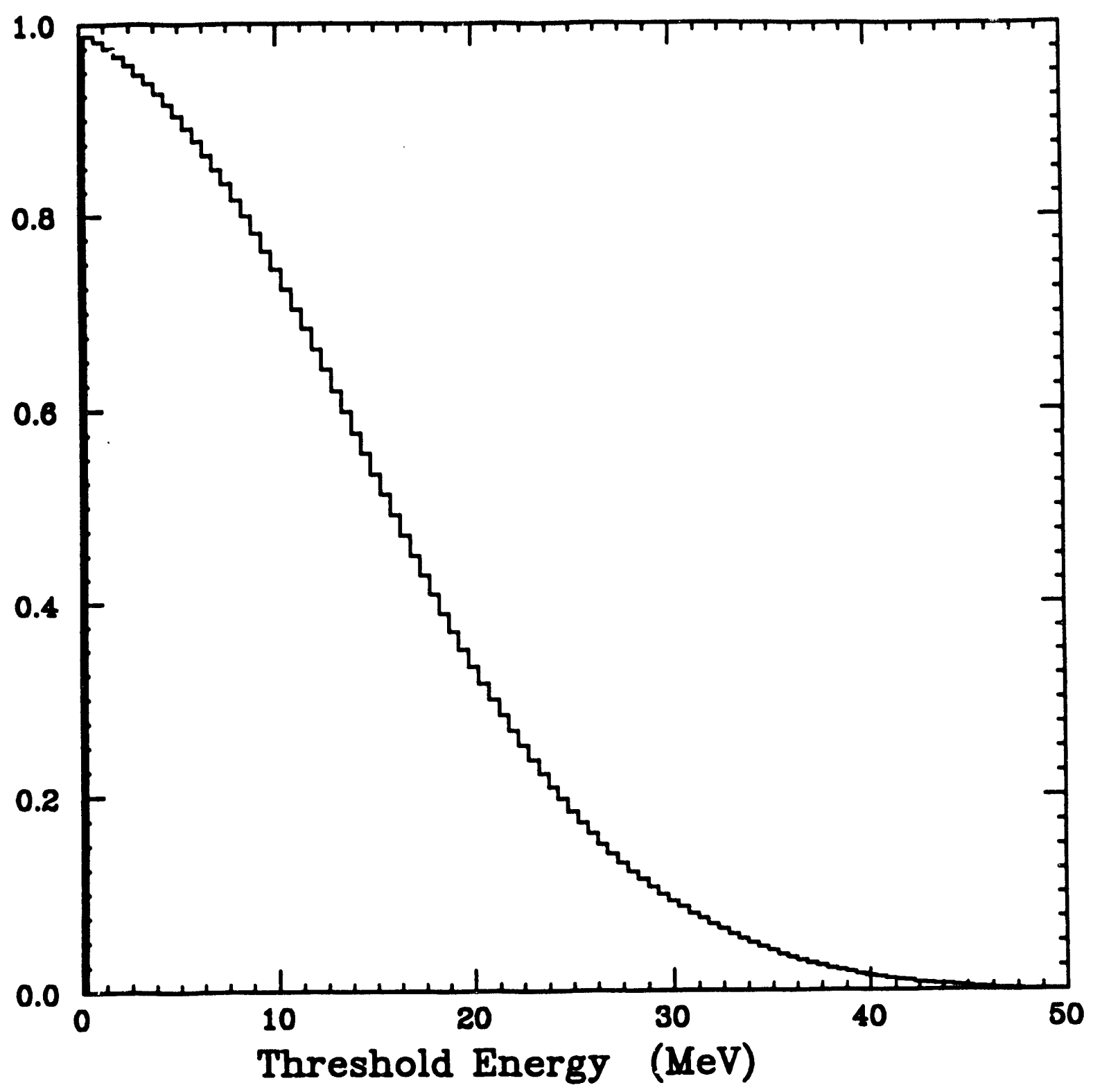

Figure 5. Integral detection efficiency of neutrino-induced positrons as a function of energy threshold. 


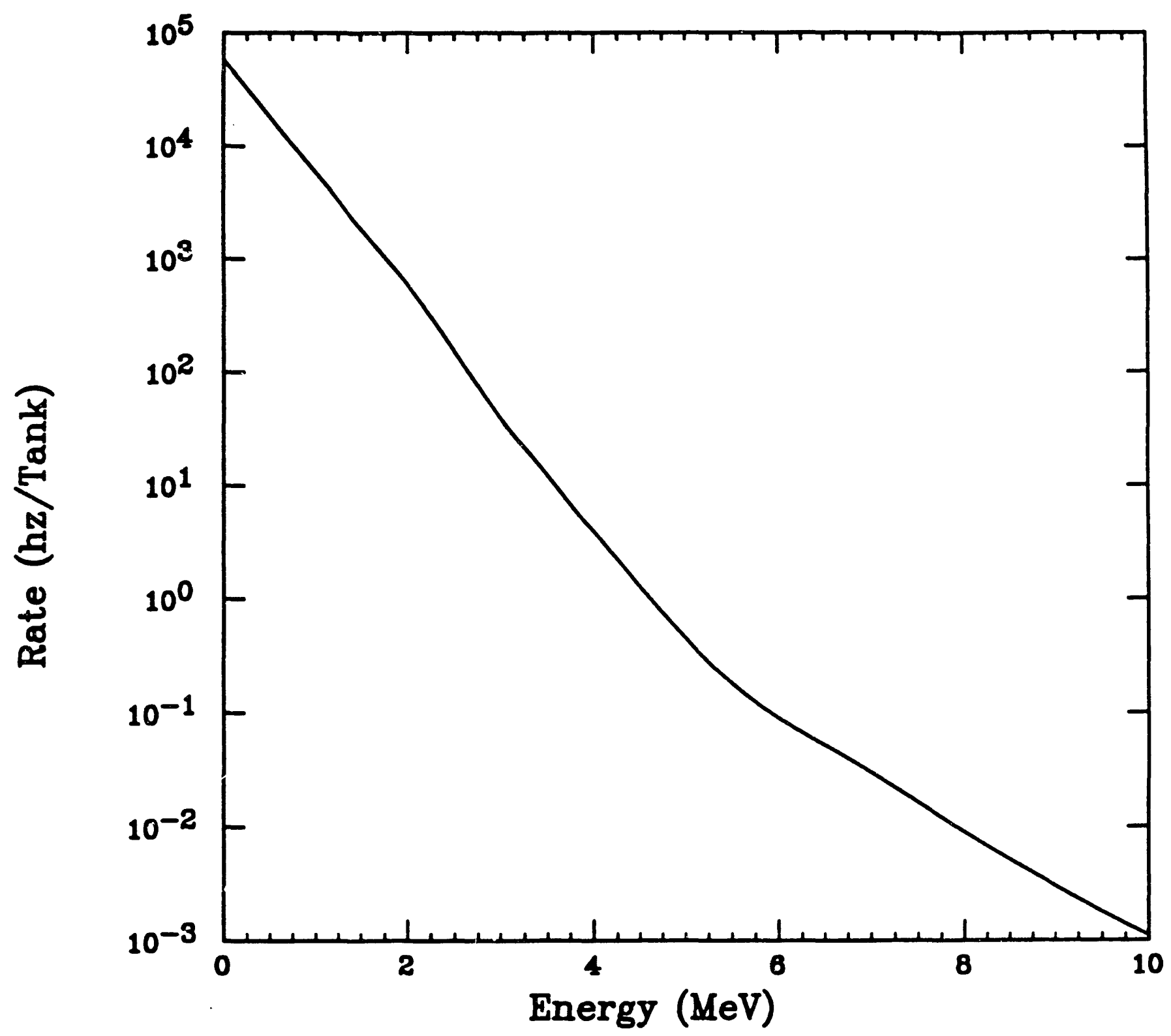

Figure 6. Integral radioactive background singles rate as a function of energy in a typical MACRO hor contal liquid scintillator module. 


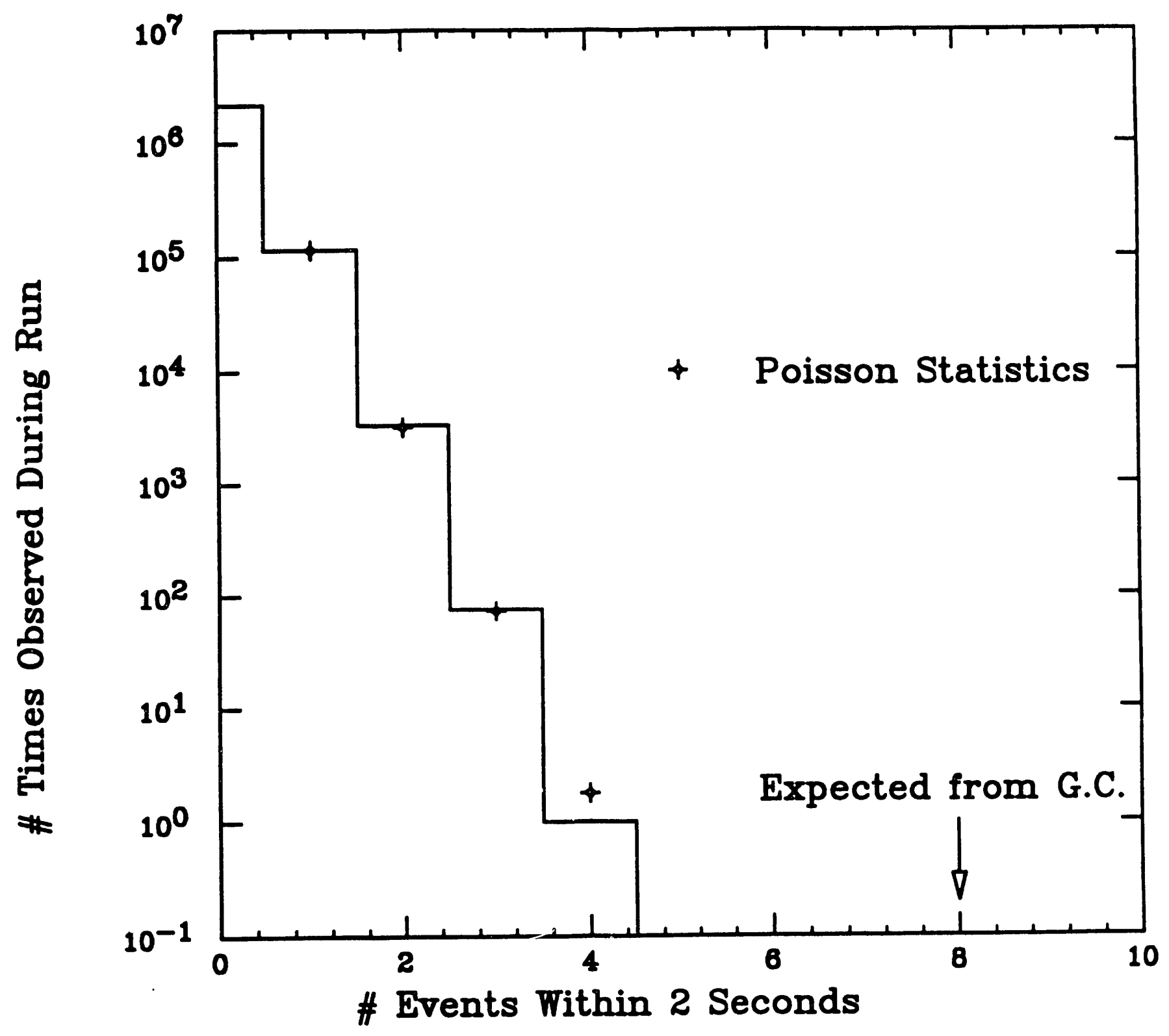

Figure 8. Result of a gravitational collapse search on the first supermodule during a three month period, showi: $g$ the number of observed 'bursts' of a given size, versus the burst size. Also shown is the expected number of events within 2 seconds from a gravitational collapse at the center of our galaxy. 


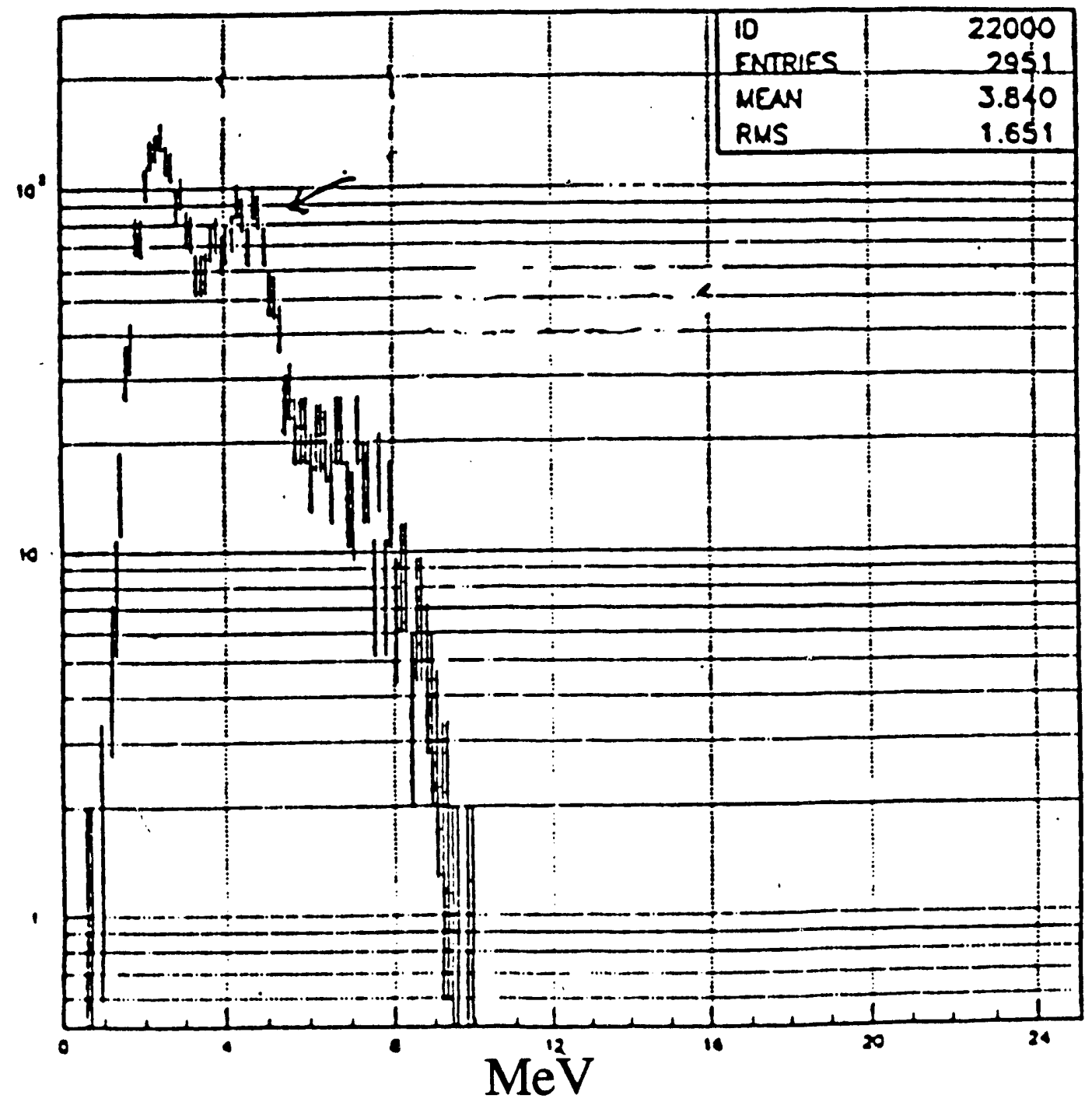

Figure 9. Energy spectrum obtained in a calibration of a MACRO scintillator tank using an $\mathrm{Am} / \mathrm{Be}$ source. 


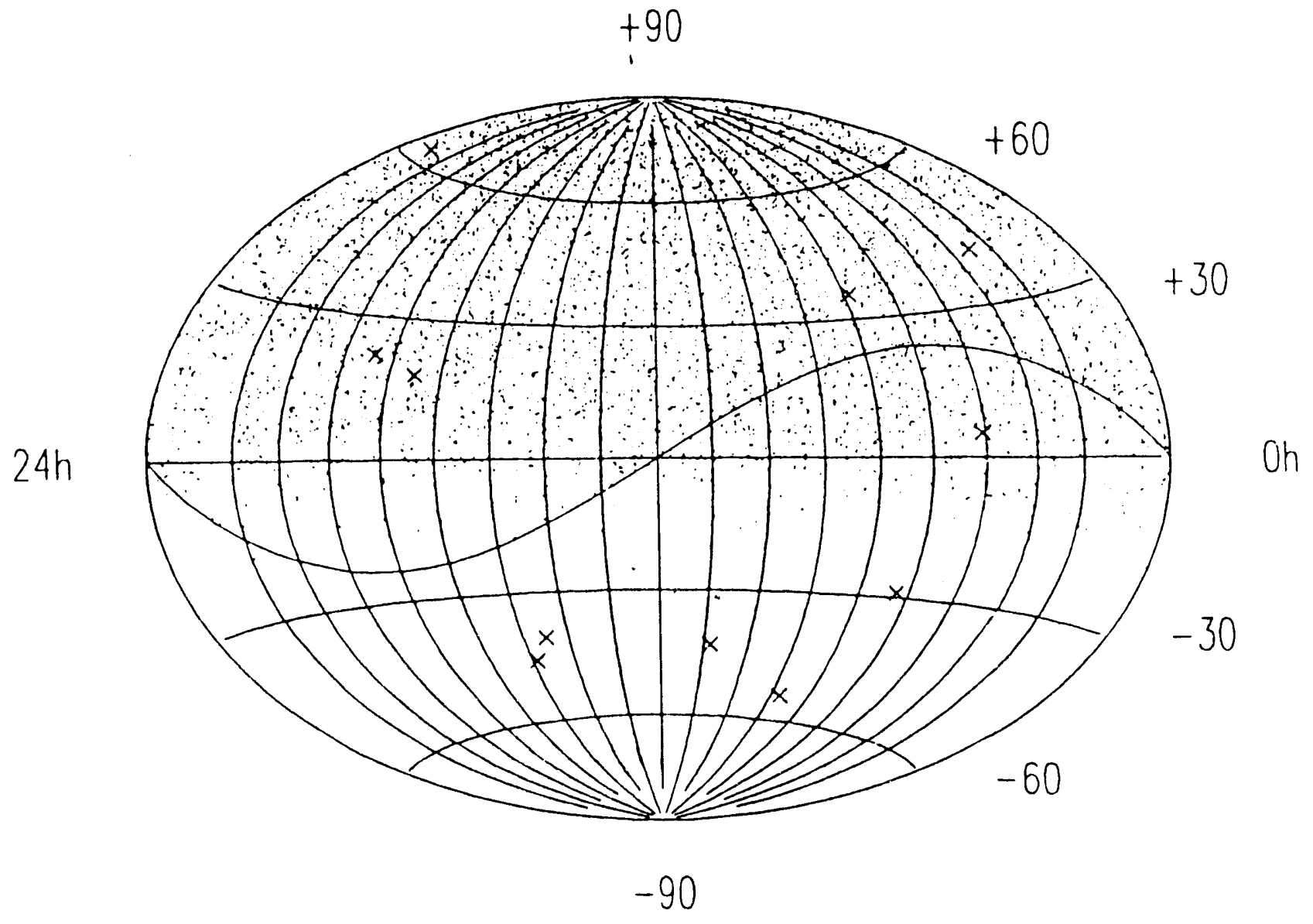

Figure 10. Distribution of muons in equatorial coordinates. 


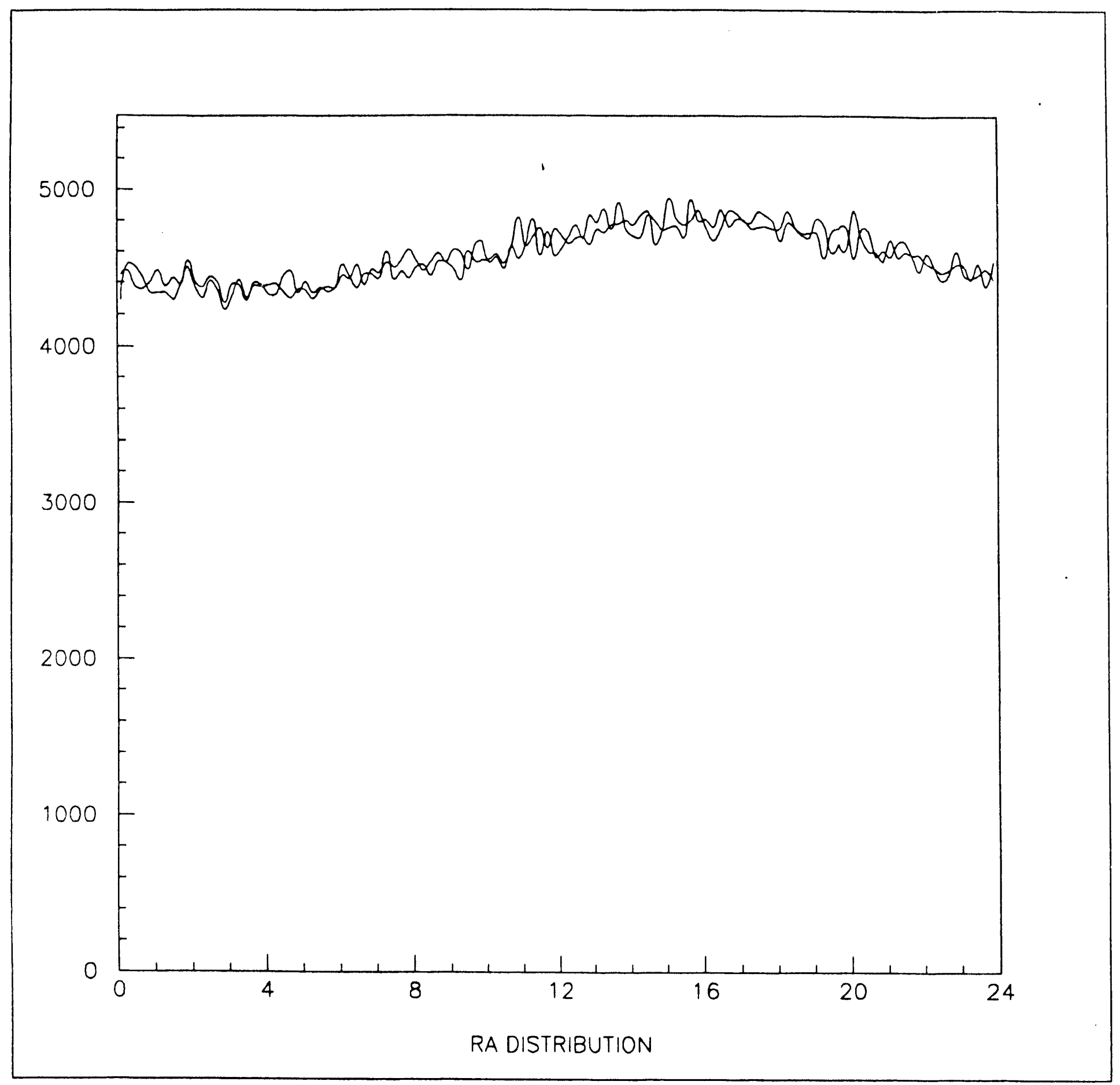

Figure 11c. The right ascension distribution with the Monte Carlo distribution superposed. The effect of live tirne is clearly seen. 


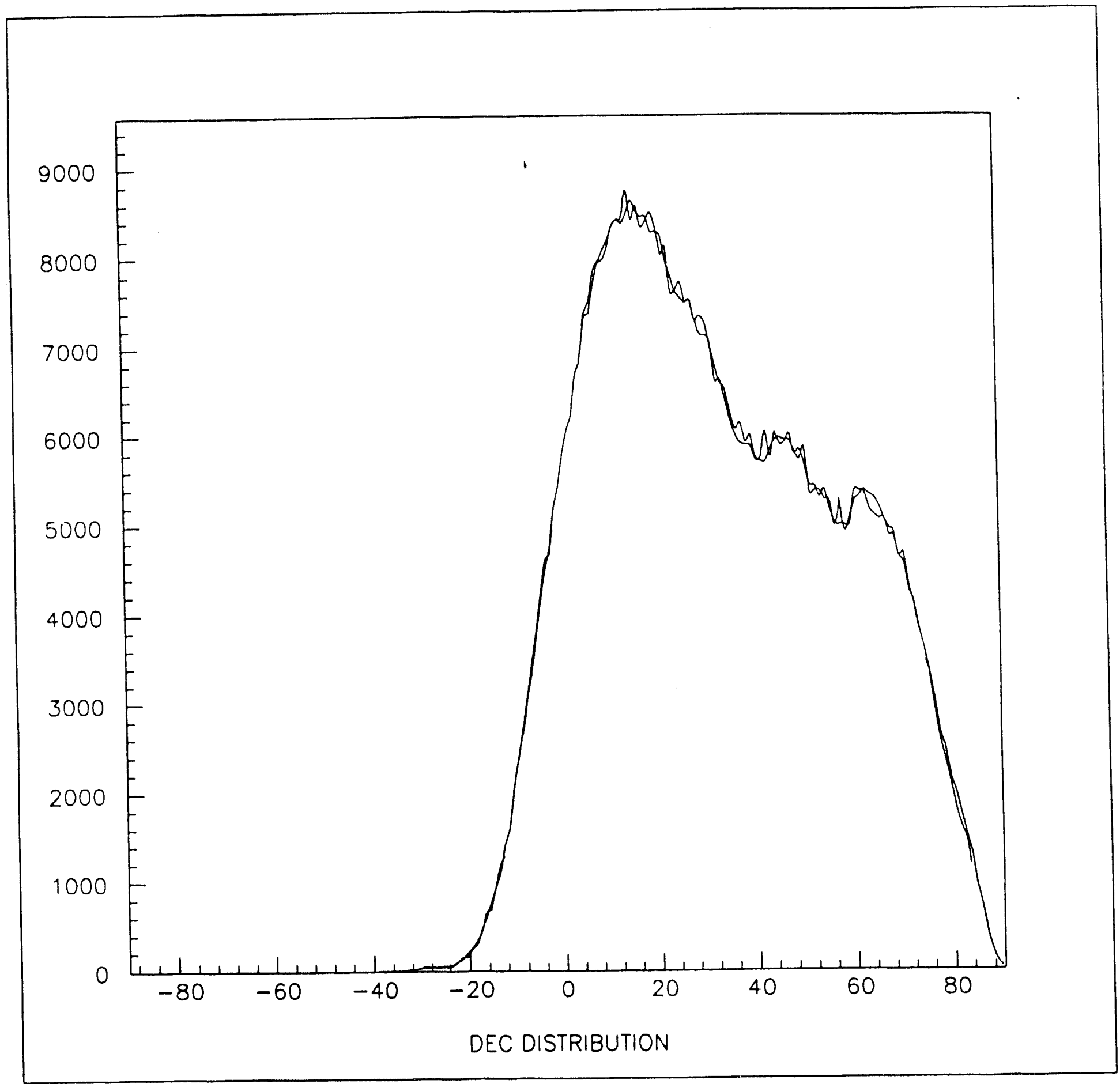

Figure 11d. Declination distribution with the Monte Carlo distribution superposed. 
10 Plones Crossed

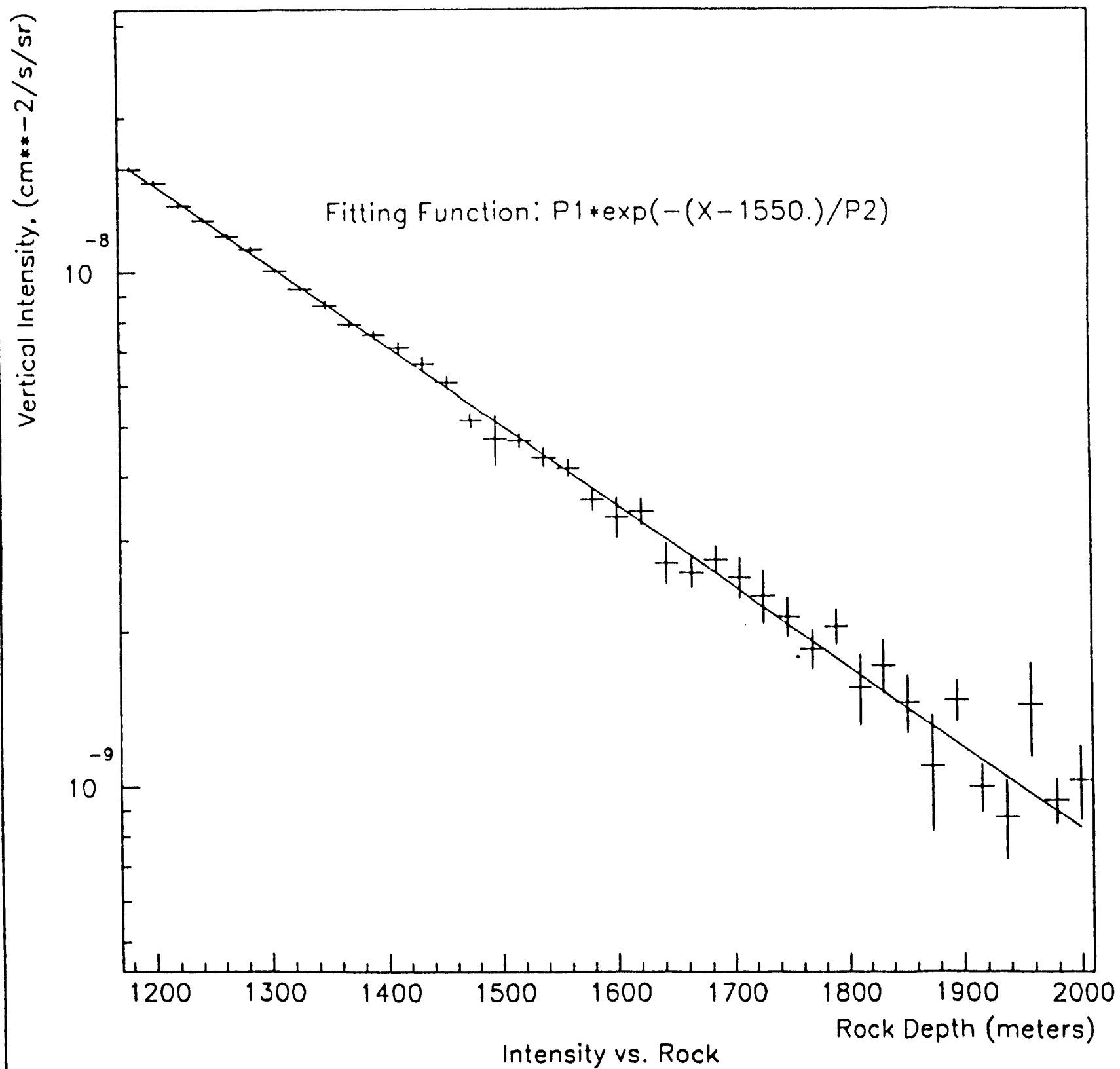

1.

Figure 12. Vertical intensity of muons vs. overburden. 


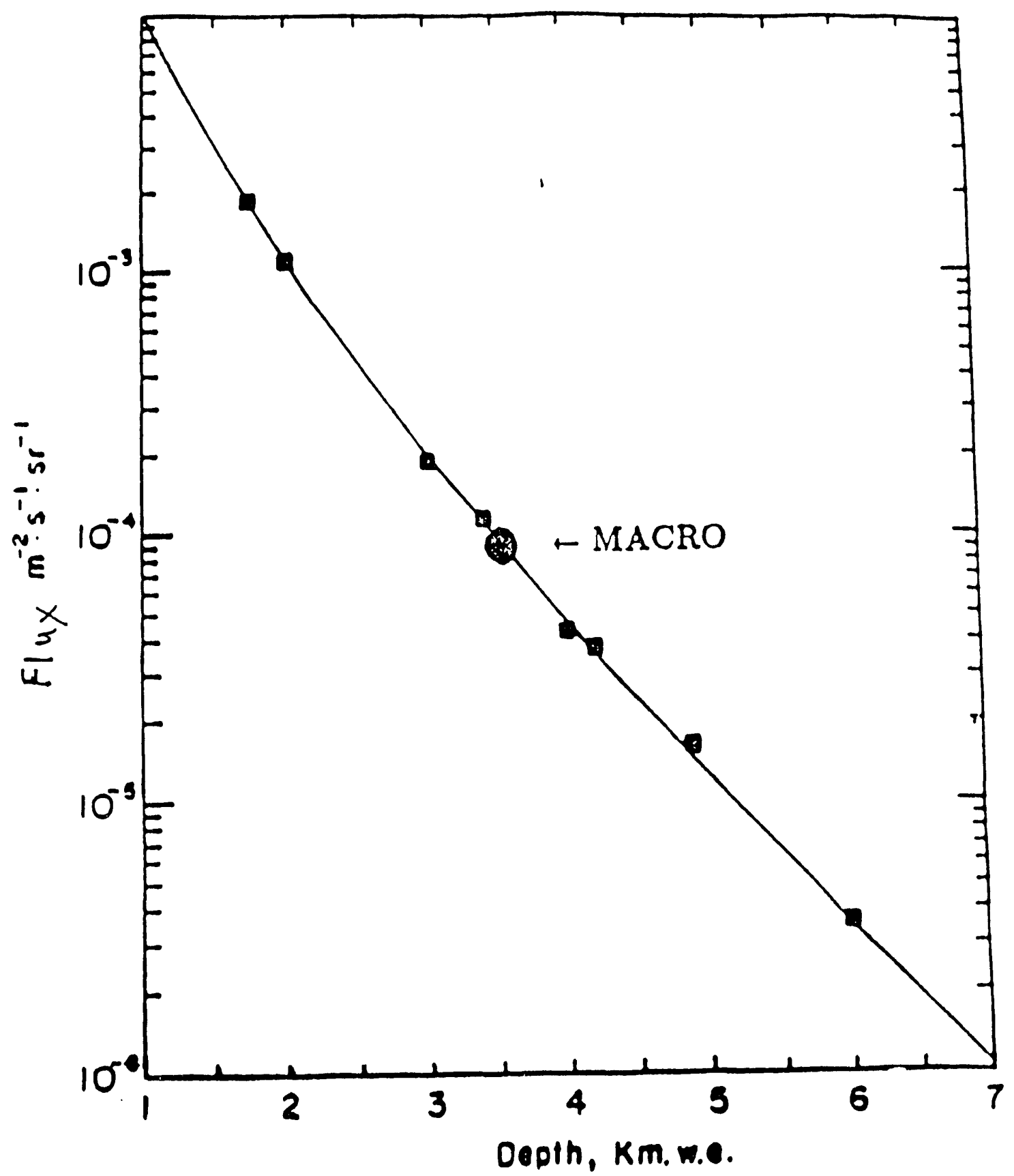

Figure 13. Muon flux as a function of depth. MACRO compared with other underground experiments. 


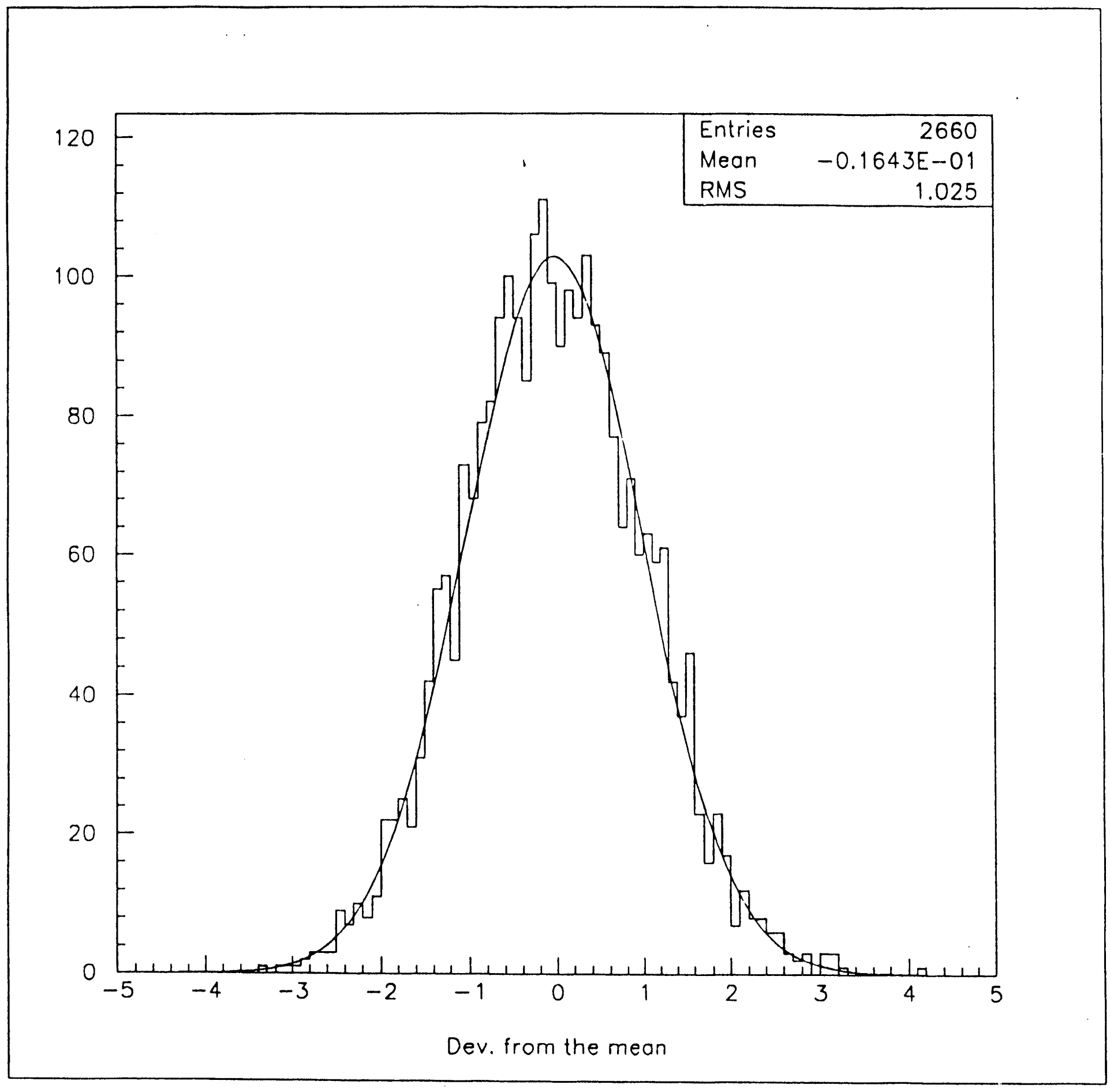

Figure 14. Search for small scale anisotropies. Plotted are deviations from the mean, $\left(N_{\left.\alpha+-N^{\infty}\right)}\right) / \sqrt{N_{\infty}}$ 


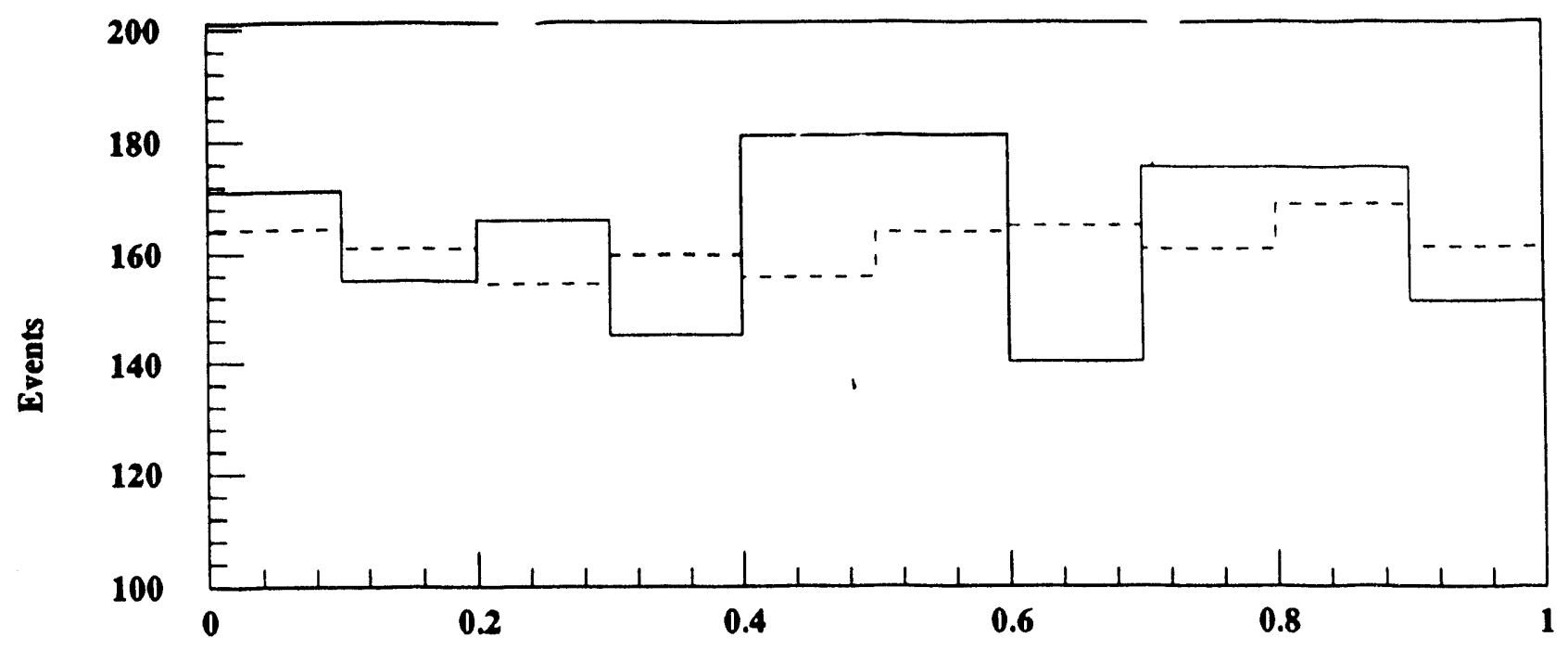

Cyg X-3 Phase

Figure 15. Phase diagram of $\mathrm{Cyg} \mathrm{X}-3$ for the complete data sample.

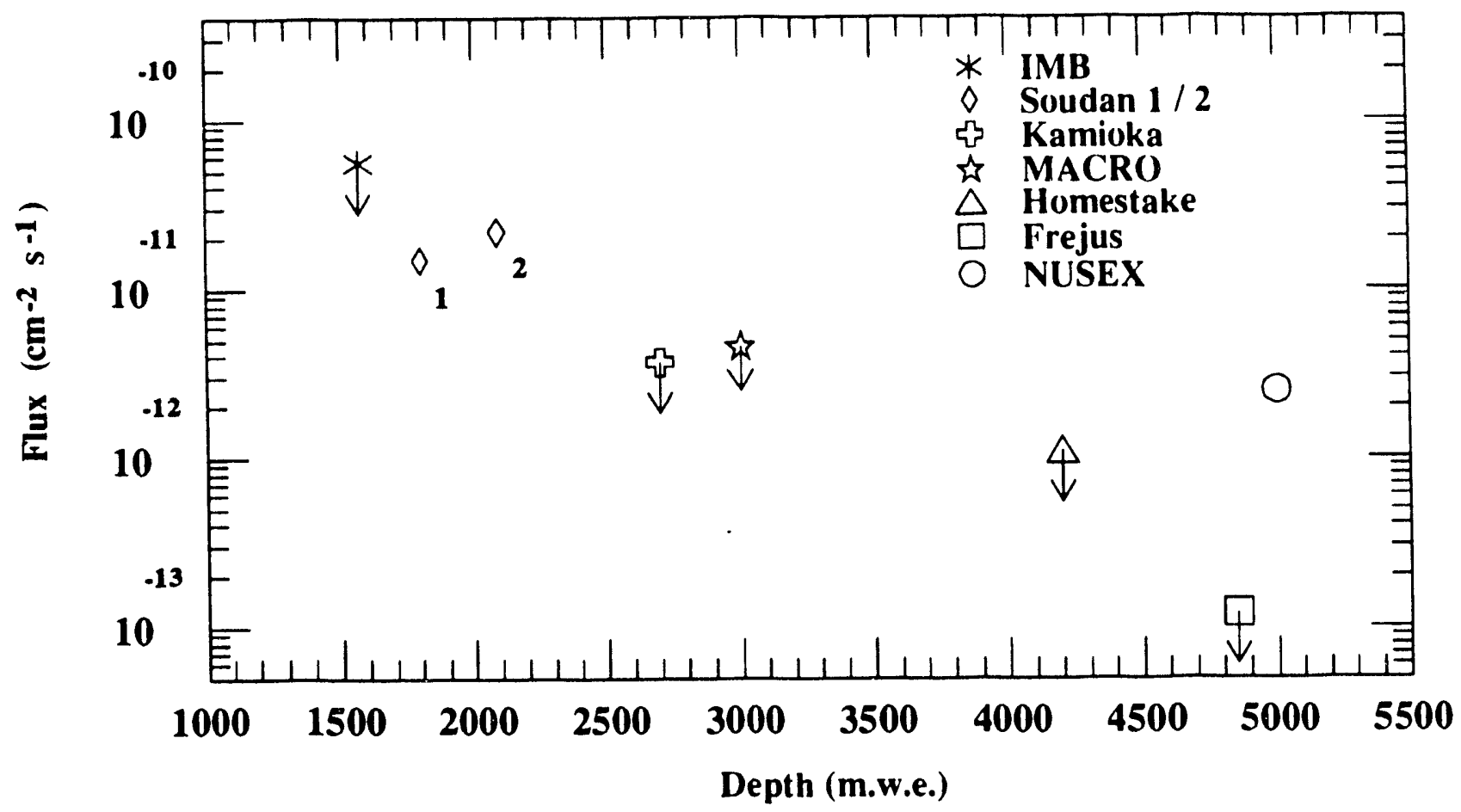

Figure 16. Measurements of the modulated muon flux from Cyg X-3. 


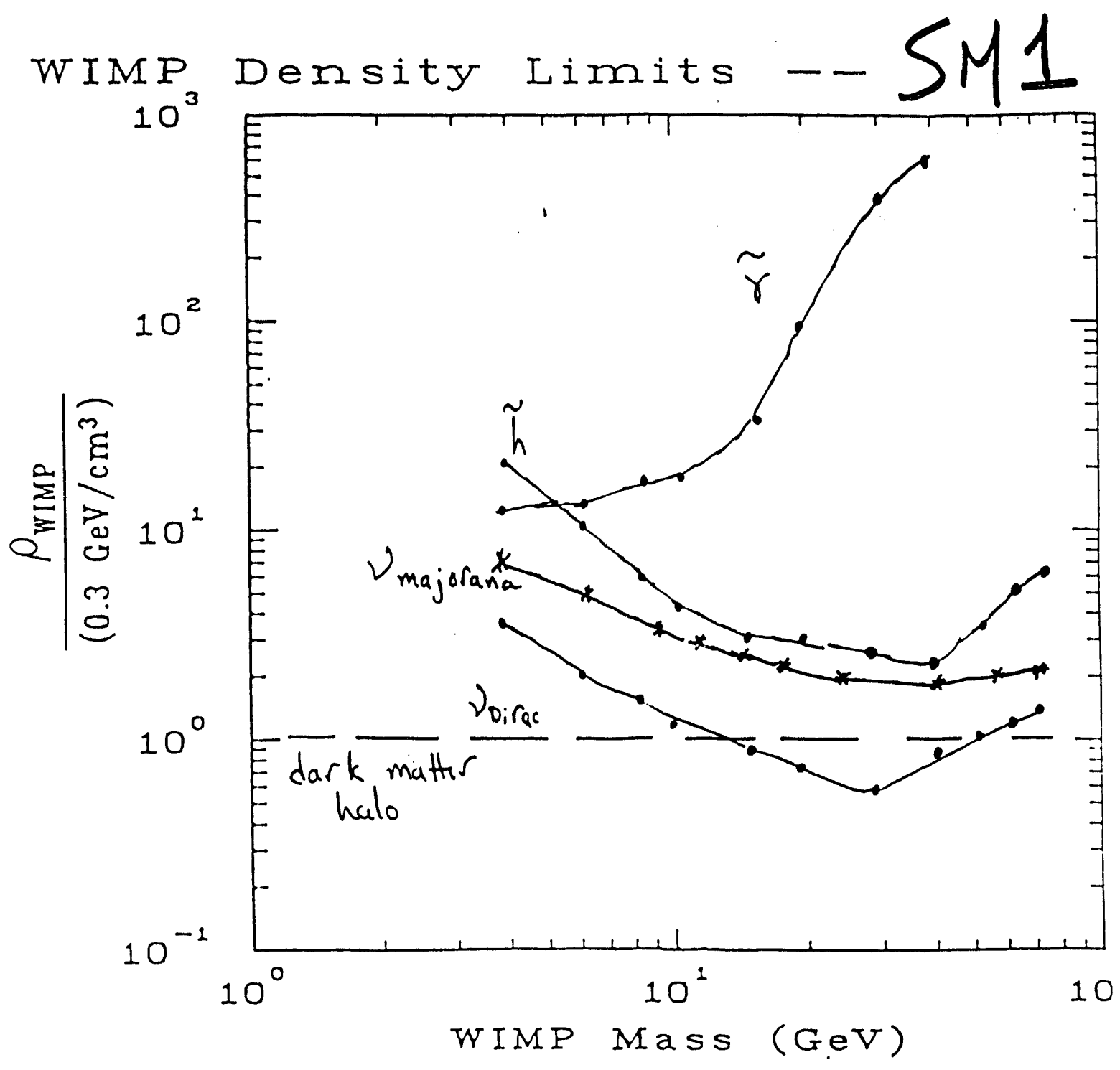

Figure 17. Upper limits to the mass density contributions to the galactic halo due to several WIMP candidates. 


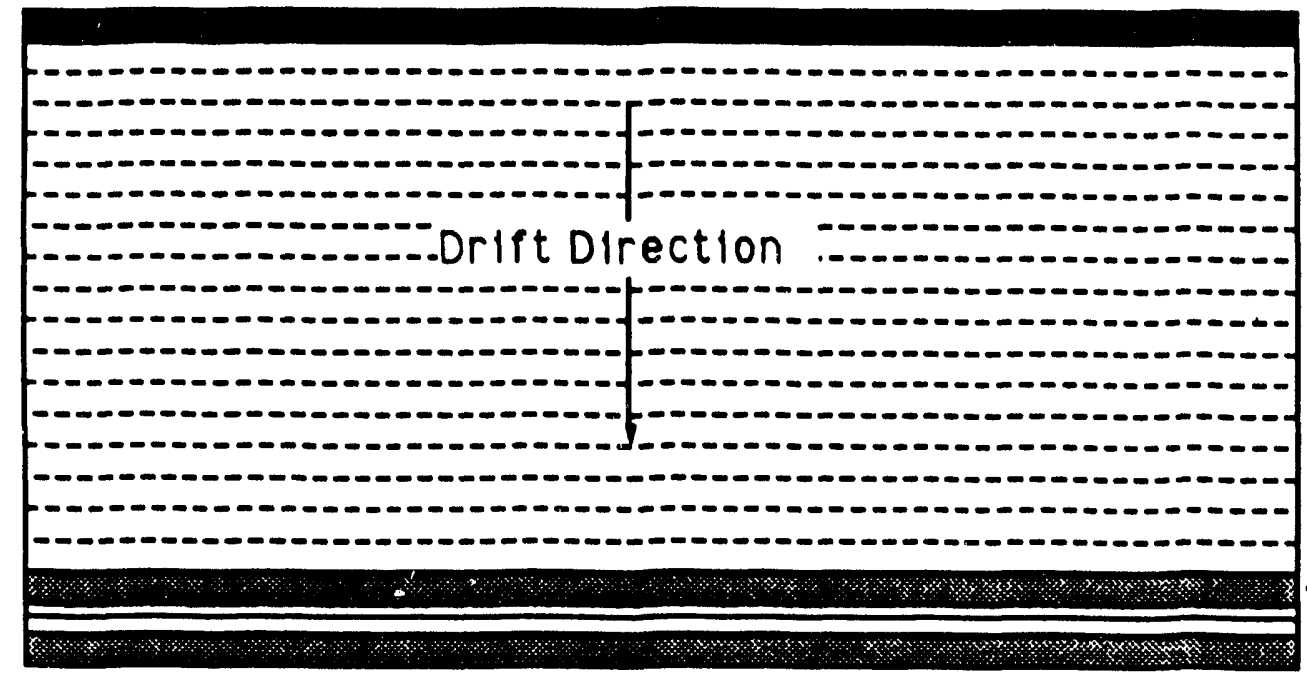

Top Vlew
Cathode Plane

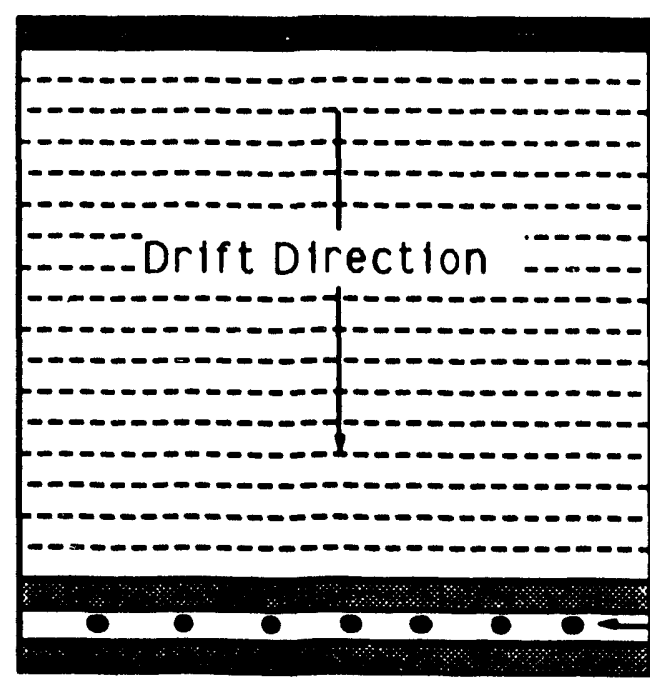

Slde Vlew
Cathode Plane
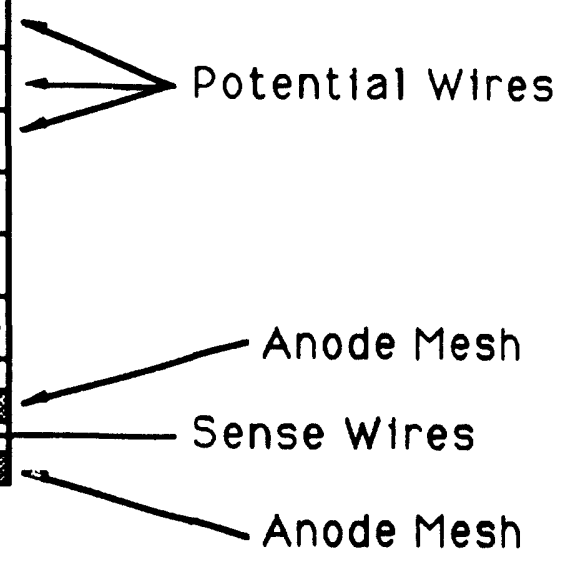

Figure 18. Drift chamber for gas studies. 


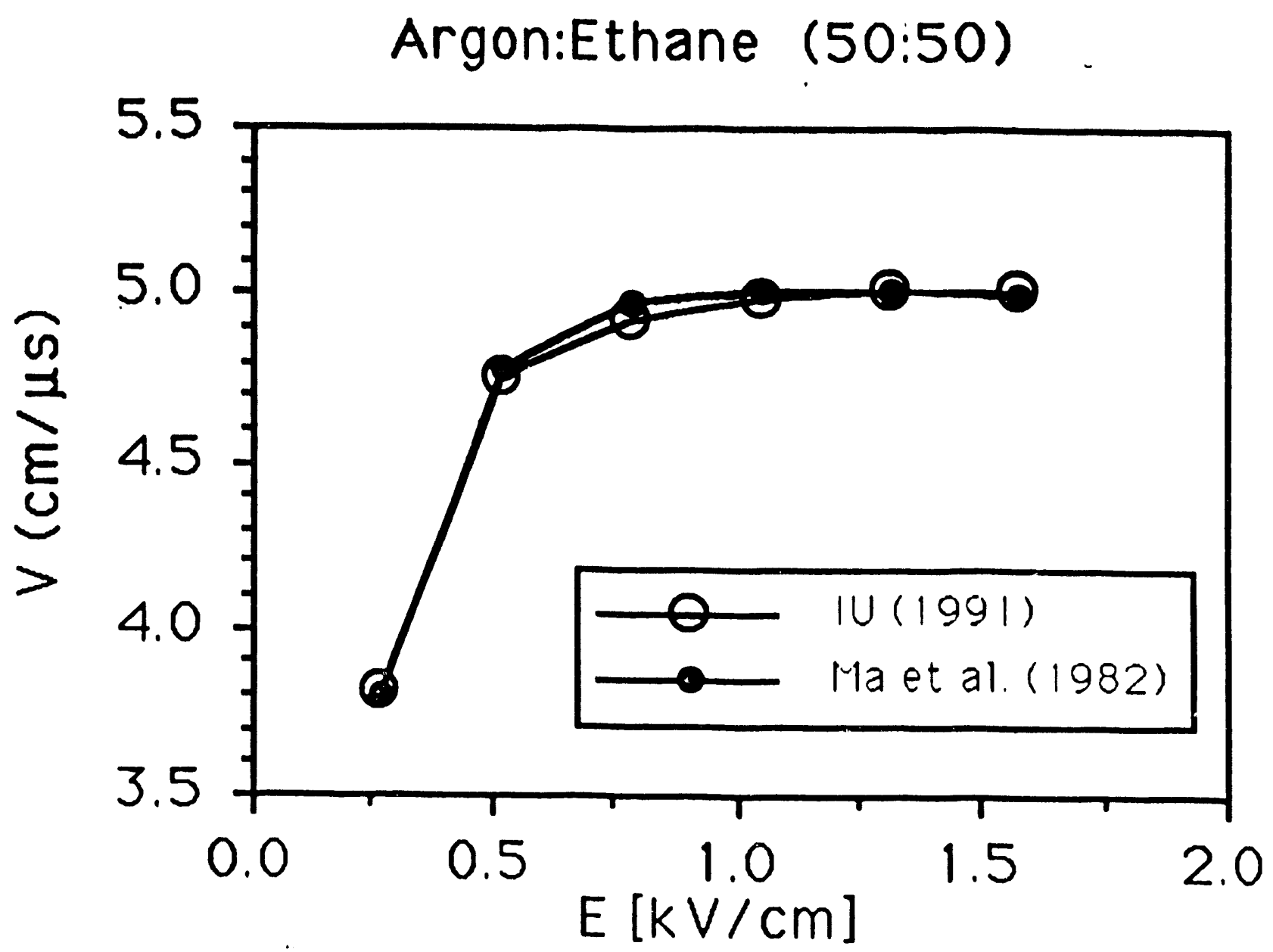

Figure 19. Drift velocity vs. electric field. 


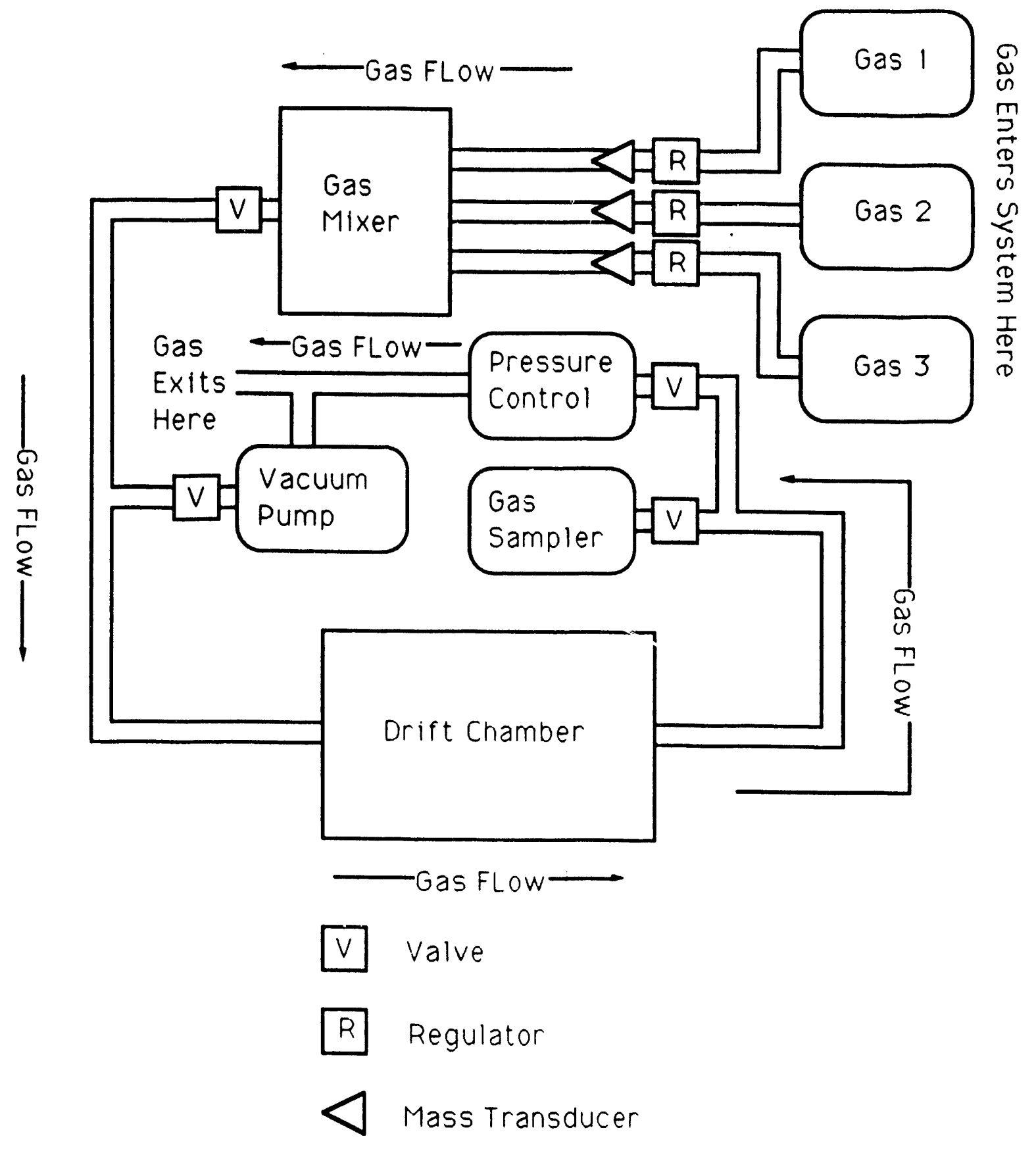

Figure 20. Drift chamber gas system. 

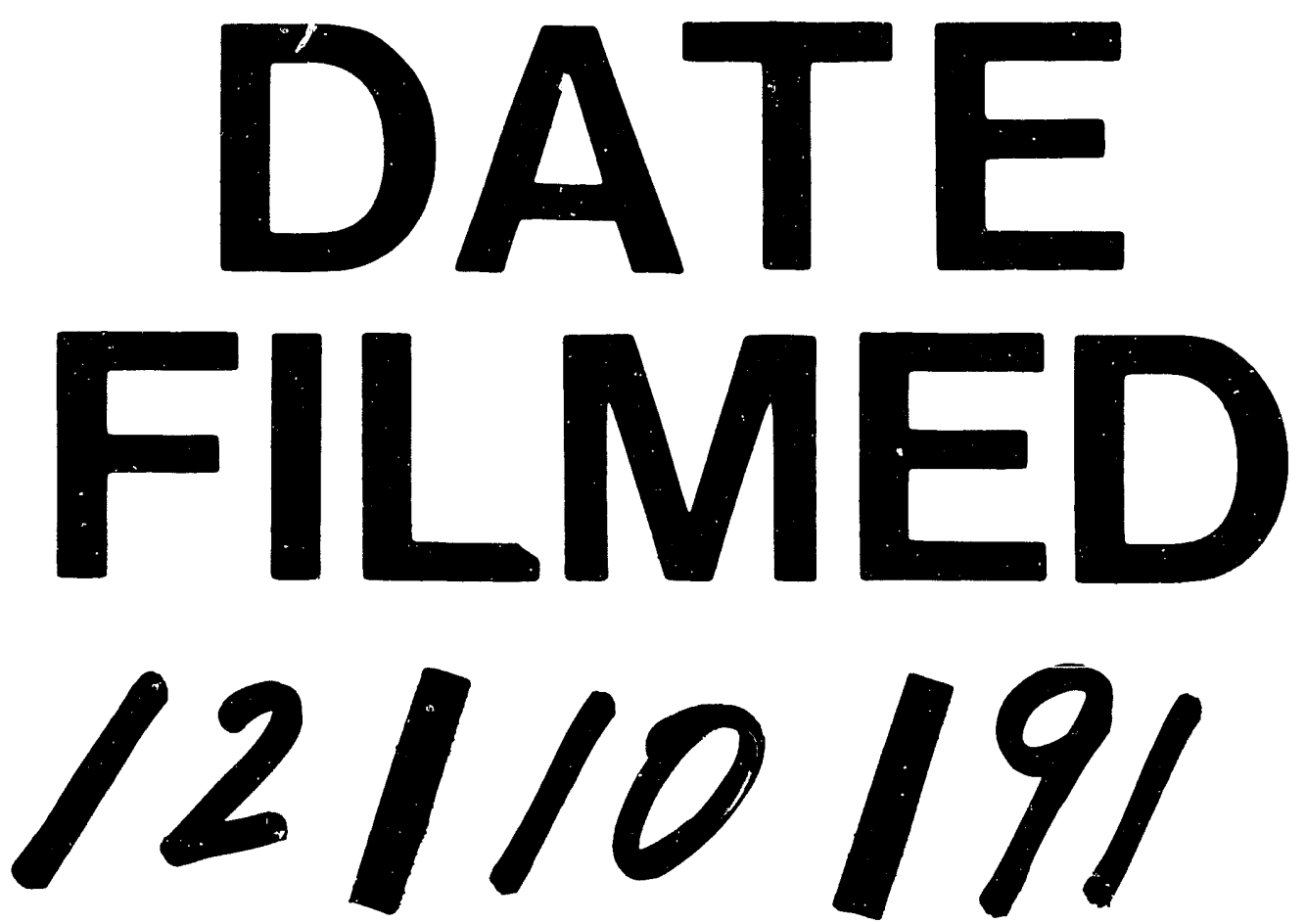
